\title{
Magnetospheric Multiscale Science Mission Profile and Operations
}

\author{
S.A. Fuselier · W.S. Lewis • C. Schiff • R. Ergun • \\ J.L. Burch · S.M. Petrinec $\cdot$ K.J. Trattner
}

Received: 14 January 2014 / Accepted: 7 August 2014 / Published online: 12 September 2014

(C) Springer Science+Business Media Dordrecht 2014

\begin{abstract}
The Magnetospheric Multiscale (MMS) mission and operations are designed to provide the maximum reconnection science. The mission phases are chosen to investigate reconnection at the dayside magnetopause and in the magnetotail. At the dayside, the MMS orbits are chosen to maximize encounters with the magnetopause in regions where the probability of encountering the reconnection diffusion region is high. In the magnetotail, the orbits are chosen to maximize encounters with the neutral sheet, where reconnection is known to occur episodically. Although this targeting is limited by engineering constraints such as total available fuel, high science return orbits exist for launch dates over most of the year. The tetrahedral spacecraft formation has variable spacing to determine the optimum separations for the reconnection regions at the magnetopause and in the magnetotail. In the specific science regions of interest, the spacecraft are operated in a fast survey mode with continuous acquisition of burst mode data. Later, burst mode triggers and a ground-based scientist in the loop are used to determine the highest quality data to downlink for analysis. This operations scheme maximizes the science return for the mission.
\end{abstract}

Keywords Magnetospheric multiscale $\cdot$ Magnetic reconnection $\cdot$ Space mission design · Spacecraft orbits

S.A. Fuselier $(\varangle) \cdot$ W.S. Lewis · J.L. Burch

Southwest Research Institute, San Antonio, TX, USA

e-mail: sfuselier@swri.edu

S.A. Fuselier

University of Texas at San Antonio, San Antonio, TX 78249, USA

C. Schiff

NASA Goddard Space Flight Center, Greenbelt, MD, USA

R. Ergun · K.J. Trattner

Laboratory for Atmospheric and Space Physics, University of Colorado, Boulder, CO, USA

S.M. Petrinec

Lockheed Martin STAR Labs, Palo Alto, CA 94304, USA 


\section{Introduction}

The MMS mission uses the Earth's magnetosphere as a laboratory to study magnetic reconnection (Burch et al. 2014, this issue]. This fundamental process converts magnetic energy into particle energy. The conversion process occurs explosively in the near-Earth magnetotail and on a quasi-steady basis at the Earth's magnetopause. The actual process of reconnection takes place in a very small region called the electron diffusion region. This region can be envisioned in three dimensions as a ribbon. It is believed to be of the order of 1-10 km thick, 10-100 km wide, and extend for tens of thousands of kilometers over the magnetopause in a continuous or nearly continuous fashion (Hesse et al. 2014, this issue; Frey et al. 2003; Fuselier et al. 2002; Trattner et al. 2007a, 2007b). In the magnetotail, the scale sizes may be a factor of about 3 times larger in thickness and width. Although it is unclear how far the reconnection region extends across the tail, it must be of the order of thousands of kilometers, similar to its extent on the magnetopause.

In the electron diffusion regions at the magnetopause and in the magnetotail, thermal electrons in the plasma become de-magnetized through a process that is yet unknown (indeed, one of the prime objectives of MMS is to discover this process). By virtue of this de-magnetization, magnetic fields "reconnect" and energy is transferred from the relaxation of the magnetic field to the plasma. The result is significant ion and electron energization, forming plasma jets that extend far from the diffusion region.

The MMS mission design is fundamentally different from other multispacecraft magnetospheric missions in that it targets this very small electron diffusion region, which the four identically instrumented spacecraft, flying in a tetrahedral configuration, probe over a range of interspacecraft separations. All aspects of the prime mission are focused on obtaining the necessary plasma, electric field, and magnetic field measurements within the region. Previous magnetospheric missions focused on broad regions in the near-Earth environment. For example, the Cluster spacecraft orbits were designed to fly through the high-latitude magnetospheric cusps to investigate plasma transfer into the Earth's magnetosphere, the THEMIS spacecraft orbits were designed to determine the timing of substorm phenomena in the magnetotail, and the recent Van Allen Probes mission was designed to investigate the Earth's radiation belts. In contrast, MMS targets very small electron diffusion regions at the magnetopause and in the magnetotail. This targeting is limited by constraints on the lift capability of the launch vehicle, the total velocity change (delta-V) that can be accommodated with on-board propulsion, and other non-science constraints (these non-science constraints are identified as "engineering" constraints in this paper).

This paper describes the MMS mission design and operations. The overall mission design and its underlying science rationale are discussed in Sect. 2. Section 3 describes the orbital characteristics by mission phase and the baseline mission requirements. Section 4 describes the design of the spacecraft configurations used to probe the science regions of interest along the magnetopause and in the tail. Section 5 describes science operations, including the burst mode design and scientist-in-the-loop (SITL) data selection. Section 6 summarizes the mission design.

\section{Science Mission Phases}

In the near-Earth environment, there are four broad regions where reconnection occurs on a frequent basis. They are the low-latitude (dayside) magnetopause, the high-latitude magnetopause tailward of the cusps, the near-Earth magnetotail, and the distant magnetotail (Science and Technology Definition Team 1999). The primary science objective of the MMS 
mission, to understand reconnection as a fundamental physical process, is achieved by investigating reconnection in two of the near-Earth regions. These two regions are the dayside magnetopause and the near-Earth magnetotail and are the two most readily accessible regions for studying reconnection. With this focus, the MMS mission was structured in two basic science mission phases, and the orbital design was optimized to maximize the probability of encountering the reconnection region in each phase.

A three-step iterative process was used by the MMS science team and the Goddard Space Flight Center Flight Dynamics group to select and optimize orbits that target the electron diffusion regions at the magnetopause and in the magnetotail. First, based on the limited available observations and modeling of the reconnection regions discussed below, the science team provided relatively loose science constraints on the MMS orbits to the Flight Dynamics group. Second, the Flight Dynamics group selected candidate orbits that met both these science constraints and a set of more stringent engineering constraints concerning eclipse duration and fuel usage. Third, the science team evaluated the candidate orbits to determine the best overall science merit. These candidate orbits were saved for further refinement through the same three-step process.

\subsection{Phase 1: Targeting the Diffusion Region on the Dayside Magnetopause}

Phase 1 targets the low-latitude dayside magnetopause. At the subsolar point, the magnetopause, distance from the Earth varies with solar wind dynamic pressure and IMF orientation and is typically between $\sim 13 R_{E}$ and $\sim 9 R_{E}$ geocentric (Sibeck et al. 1991). Phase 1 consists of two dayside sub-phases (Phases 1a and 1b) separated by a transitional sub-phase on the night side (Phase 1x). Assessing the scientific merit of candidate Phase 1 orbits requires knowledge of the statistical location of the reconnection line at the magnetopause. However, establishing the statistical location of the reconnection line is difficult using data from spacecraft magnetopause crossings (see, e.g., Fuselier and Lewis 2011) because it is difficult to determine the location of the reconnection line except when a spacecraft encounters it. These encounters are rare (e.g., Phan et al. 2003), and there are no good statistics on the location of reconnection at the magnetopause from these individual encounters. Fortunately, the location of the reconnection line can be determined using observations in the Earth's magnetospheric cusps (Fuselier et al. 2000). From a large number of cusp observations, an empirical model of the reconnection line location was developed (Trattner et al. 2007a, 2007b) for conditions when the interplanetary magnetic field (IMF) was southward $\left(B_{z}<0\right)$. This model uses a 3-D magnetopause shape and draping of the IMF (including bending of the field across the shock) to compute the shear in the magnetic field at all points on the magnetopause. The location of reconnection is empirically related to regions of high and intermediate shear. The empirical model has been validated in several independent tests (Fuselier et al. 2011; Petrinec et al. 2011; Dunlop et al. 2011; Trattner et al. 2012). The model predicts the location of the reconnection line using the interplanetary magnetic field (IMF) orientation.

Figure 1 shows the predicted location of the reconnection line for three IMF orientations (top to bottom, these orientations are $B_{y}$ dominant, $\left|B_{z}\right|$ dominant, and $B_{x}$ dominant, with $B_{z}<0$ for all three orientations). In each panel, the magnetic shear angle (i.e., the angle between the magnetic field in the magnetosheath and in the magnetosphere at the model magnetopause boundary) projected into the $Y-Z$ plane is color coded with red showing high shear $\left(>150^{\circ}\right)$ and purple showing low shear $\left(<50^{\circ}\right)$. Anti-parallel reconnection lines occur where the magnetosheath and magnetospheric fields are exactly opposed (i.e., anti-parallel with shear angle $=180^{\circ}$ ). The circle is the projected location of the terminator (where 
Fig. 1 Empirical model for the location of the reconnection line for three different solar wind IMF conditions. Each panel shows a view from the Sun of the color-coded shear angle between the magnetosheath and magnetospheric magnetic fields. Red regions are where the shear is the highest (up to $180^{\circ}$ ). During $B_{y}$ dominant conditions (top panel), the reconnection line follows the anti-parallel reconnection region from the dawnside to near the cusp. Before arriving at the cusp, the reconnection line cuts across the dayside magnetopause, crossing the noon-midnight meridian just north of the subsolar point before joining the anti-parallel reconnection region on the duskside. For $\left|B_{z}\right|$ and $B_{x}$ dominant conditions, the reconnection line follows the anti-parallel regions up to the cusps and there is a break in the reconnection line at the noon-midnight meridian
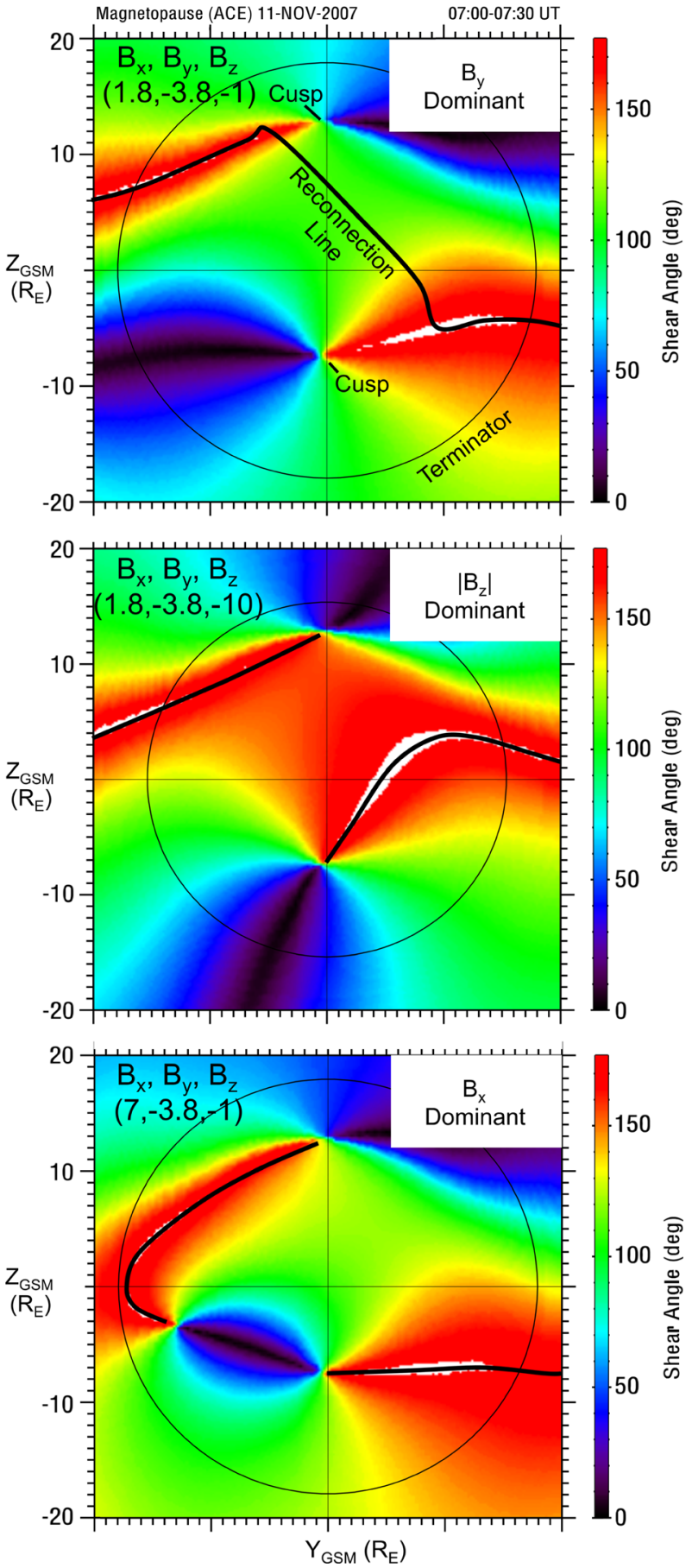
$X=0$ ) and, in the top panel, the locations of the magnetospheric cusps in the northern and southern hemispheres are indicated.

The top panel of Fig. 1 shows the reconnection line location for northern hemisphere winter (November) when the IMF is southward $\left(B_{z}<0\right)$ and $B_{y}$ is dominant. Specifically, this panel shows the reconnection line when the IMF clock angle $\left(\arctan \left(B_{y} / B_{z}\right)\right.$, measured from $0^{\circ}-360^{\circ}$ ) is $255^{\circ}$. The reconnection line in the top panel in Fig. 1 is qualitatively similar to the one that occurs when the IMF clock angle is between $90^{\circ}$ and $155^{\circ}$ or $205^{\circ}$ and $270^{\circ}$. Under these conditions, the reconnection line stretches across the entire dayside magnetopause from dawn (left) to dusk (right). From the dawnside, it follows the anti-parallel shear location but, at some point approaching the northern cusp, it deviates from the anti-parallel location and cuts across the dayside magnetopause north of the subsolar point (where $Y=Z=0$ ). It eventually joins the other anti-parallel location in the southern hemisphere and follows this location out past the terminator on the dusk side. The top panel in Fig. 1 shows the reconnection line for $B_{y}<0$. If $B_{y}>0$, then the reconnection line orientation mirrors about the noon-midnight meridian $(Y=0)$. The angle the reconnection line makes with respect to the $Y=0$ axis depends on the relative magnitudes of $B_{y}$ and $B_{z}$.

The location of the reconnection line relative to the subsolar point is determined by season (i.e., by dipole tilt). For northern hemisphere winter, the reconnection line passes north of the subsolar point as illustrated in the top panel of Fig. 1. For the equinoxes, the reconnection line passes through the subsolar point. For northern hemisphere summer, it passes south of the subsolar point.

The middle panel of Fig. 1 shows the reconnection line location for northern hemisphere winter (November) when the IMF is southward $\left(B_{z}<0\right)$ and $B_{z}$ is dominant. Specifically, this panel shows the reconnection line when the IMF clock angle is $208^{\circ}$. This reconnection line is qualitatively similar to those that occur when the IMF clock angle is between $155^{\circ}$ and about $205^{\circ}$. Under these conditions, the reconnection line follows the anti-parallel location from the dawn terminator up to the cusp. There is a break in the line at the noonmidnight meridian ( $Y=0$ in Fig. 1), and the reconnection line follows the anti-parallel location from the southern cusp to the dusk terminator. As the clock angle approaches $180^{\circ}$ (exactly southward IMF), the anti-parallel region shifts closer to the subsolar point. At $180^{\circ}$, the reconnection line is nearly parallel to the geomagnetic equator $(Z=0$ line) right up to the noon-midnight meridian. At that point, the reconnection line becomes more vertical as it connects to the northern and southern cusps. Similar to the reconnection line for $B_{y}$ dominant conditions, the location of the reconnection line relative to the geomagnetic equator for $\left|B_{z}\right|$ dominant conditions depends on season. For northern hemisphere winter, the reconnection line parallels the geomagnetic equator in the northern hemisphere $(Z<0)$ as shown in the middle panel of Fig. 1. For the equinoxes, the reconnection line is at the geomagnetic equator, and for northern hemisphere summer, the line parallels the geomagnetic equator in the southern hemisphere $(Z>0)$.

The bottom panel of Fig. 1 shows the reconnection line for northern hemisphere winter (November) when the IMF is southward $\left(B_{z}<0\right)$ and $B_{x}$ is dominant. Specifically, this panel shows the reconnection line when the $B_{x} /|B|>0.9$ and is representative of the reconnection line when $\left|B_{x}\right| /|B|>0.7$. Under these conditions, the reconnection line is similar to that for $B_{z}$ dominant conditions in that it follows the anti-parallel location from the dawn and dusk terminators up to the northern and southern hemisphere cusps, respectively. There is a break in the reconnection line at the noon-midnight meridian ( $Y=0$ in Fig. 1), and the reconnection line follows the anti-parallel location from the southern cusp.

For MMS mission planning, the maximum shear reconnection model of Trattner et al. (2007a, 2007b) (Fig. 1) provides a relatively simple way to assess the targeting of the 
Table 1 Predicted number of encounters with the maximum shear model reconnection diffusion region as a function of MMS mission phase for the 15 March 2015 launch

\begin{tabular}{llll}
\hline $\begin{array}{l}\text { Mission } \\
\text { Phase }\end{array}$ & $\begin{array}{l}\text { \# of MP crossings } \\
\text { (all IMF orientations) }\end{array}$ & $\begin{array}{l}\text { \# of crossings within } 0.5 R_{E} \\
\text { of the reconnection line }\end{array}$ & $\begin{array}{l}\text { Percentage of crossings within } \\
0.5 R_{E} \text { of the reconnection line }\end{array}$ \\
\hline 1a & 1496 & 47 & $3.1 \%$ \\
1b & 1068 & 16 & $1.5 \%$ \\
Total & 2564 & 63 & $2.5 \%$ \\
\hline
\end{tabular}

reconnection diffusion region and the scientific merit of a particular set of MMS orbits. Using the Tsyganenko 1996 semiempirical model magnetosphere and magnetopause (Tsyganenko 1995) and the solar wind conditions (as observed by the ACE spacecraft) one solar cycle prior to the MMS mission timeline, the MMS science team and the GSFC Flight Dynamics analysis group predicted the number of expected magnetopause crossings (see Fig. 2). The predictions include all standard orbit perturbations over the two-year prime mission. With IMF data for each crossing, the distance from the spacecraft to the maximum shear model reconnection line is determined. Then, the number of crossings within a prescribed distance $\left(0.5 R_{E}\right)$ to the model reconnection line provides a quantitative measure of the targeting of the reconnection region.

Figures 2 and 3 show the Phase 1a and 1b orbits for a nominal 15 March 2015 MMS launch date and the predicted magnetopause encounters (red dots). There are multiple crossings during each orbit due to the inward and outward motion of the (model) magnetopause in response to variable solar wind conditions. Table 1 lists the number of predicted crossings (1224 in Phase 1a and 813 for the shorter Phase 1b) and the number and percentage of crossings that pass within $0.5 R_{E}$ of the model reconnection line as a function of mission phase for the nominal launch date. These results are typical of other launch dates and are independent of the phase of the solar cycle (Griffiths et al. 2011). Although no statistical survey of reconnection region encounters has been performed, the predicted magnetopause crossings are an indication of the probability that MMS will encounter an electron diffusion region. The encounter probabilities of a few percent given in Table 1 (see also Griffiths et al. 2011) are consistent with the relatively rare encounters of the reconnection line by missions such as Cluster and THEMIS (e.g., Phan et al. 2003; Trattner et al. 2012).

The probability of encountering the reconnection diffusion region near the subsolar point is higher than surrounding magnetopause regions (except for the magnetospheric cusps) (Griffiths et al. 2011) because, as shown in the top panel of Fig. 1, when IMF $B_{y}$ is dominant, the reconnection line passes near the subsolar point. At Earth, the IMF is typically in the Parker spiral orientation, with $B_{y} \approx-B_{x}$ and the magnitude of $B_{z}$ typically less than that of $B_{y}$ or $B_{x}$. Therefore, reconnection lines with characteristics similar to the one in the top panel of Fig. 1 are more common than the reconnection lines with characteristics similar to those in the middle and bottom panels of Fig. 1. Owing to the seasonal variation in the dipole tilt angle noted above, for the nominal March launch date (December magnetopause encounter), it is advantageous to have the orbit apogee encounter the noon meridian magnetopause somewhat above the subsolar point.

Figure 4 shows probability maps for encountering the reconnection diffusion region at the dayside magnetopause. The maps are similar to those in Griffiths et al. (2011), but use the predicted orbits for the 15 March 2015 launch date and the predicted magnetopause crossings in Figs. 2 and 3. As illustrated in Fig. 4, the magnetopause crossings (plus signs) 
Fig. 2 Predicted Phase 1a orbits for the 15 March 2015 launch.

The orbits are projected onto the Geocentric Solar Magnetospheric (GSM) $X-Y$ plane with the Sun to the left (top panel) and onto the GSM $Y-Z$ plane as viewed from the Sun (bottom panel). GSM coordinates are used throughout this paper, unless otherwise stated. The black curve indicates the nominal location of Earth's magnetopause. The black oval shows a representative Phase 1a orbit. The green region shows the overlay of all the individual Phase 1a orbits as the orbit apogee passes through the dayside magnetosphere and magnetopause from the start of science operations on the dusk side of the Earth, through the subsolar point, and to the dawn side (over a period of approximately 6 months from XX 2015 to YY 2015). The red dots identify predicted magnetopause crossings and are used to make predictions of the probability of encountering an electron diffusion region. During this phase, there are 1581 predicted magnetopause crossings, including a number near the subsolar region, where the probability of encountering the reconnection diffusion region is highest
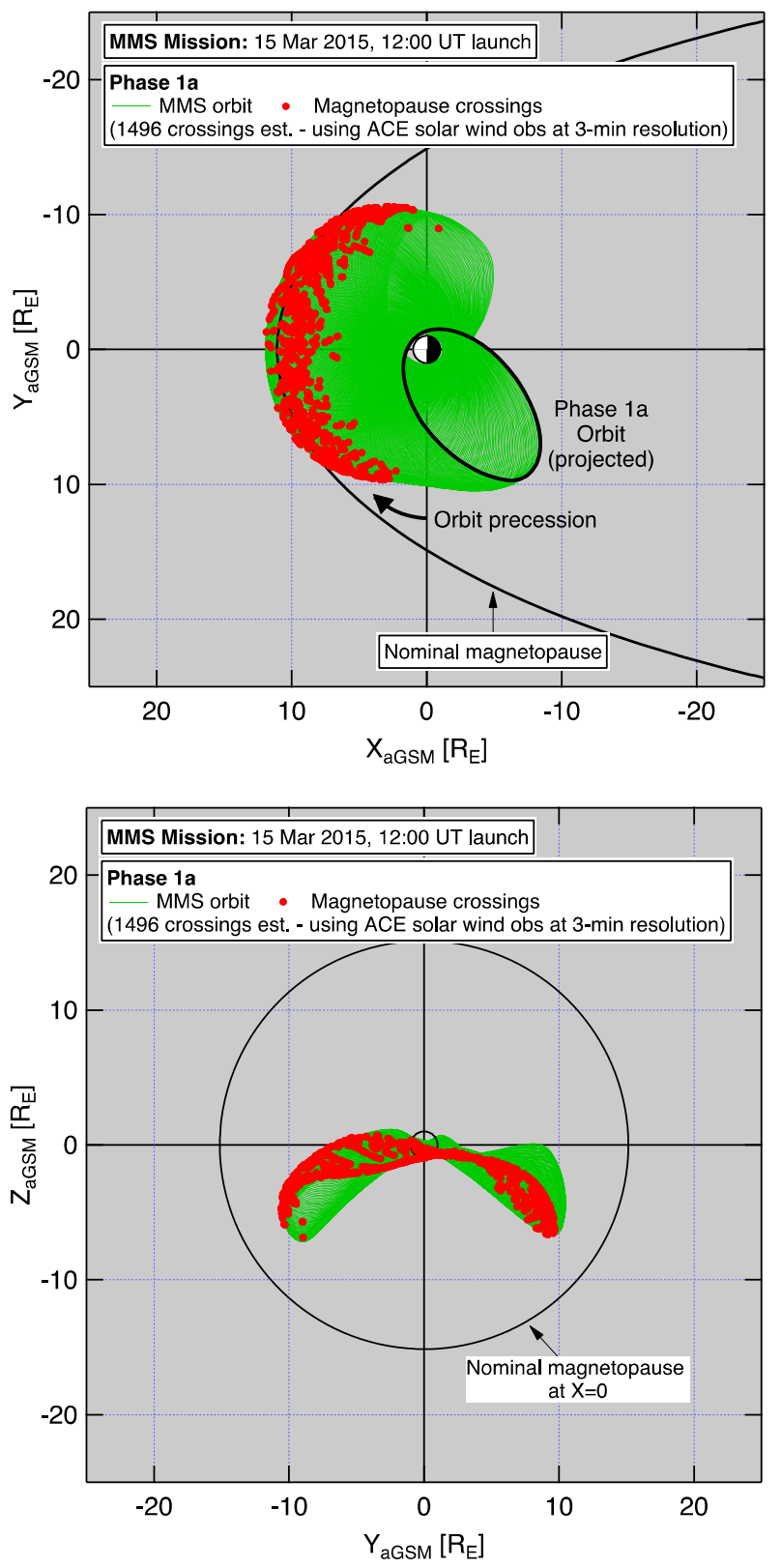

roughly follow the regions at low latitude where the predicted probabilities of encountering the diffusion region are high.

\subsection{Phase $1 x$}

Phase $1 \mathrm{x}$ is a transitional phase during which the orbit passes from dawn to dusk (from 06:00 to 18:00 GSE local time) through the near-Earth magnetotail. With a $12-R_{E}$ apogee, 
Fig. 3 Predicted Phase $1 b$ orbits shown in the same format as in Fig. 2. Phase $1 \mathrm{~b}$ follows the 180-day transitional phase (Phase 1x) during which the line of apsides passes through the magnetosphere from dawn (end of Phase 1a) back to dusk (see Sect. 3). During Phase 1b, the line of apsides passes through the magnetosphere from dusk through the subsolar region to about 10:00 GSE local time. Fewer magnetopause crossings (813) are predicted than for Phase 1a because Phase $1 \mathrm{~b}$ ends early to allow time for orbit apogee raising during Phase $2 \mathrm{a}$
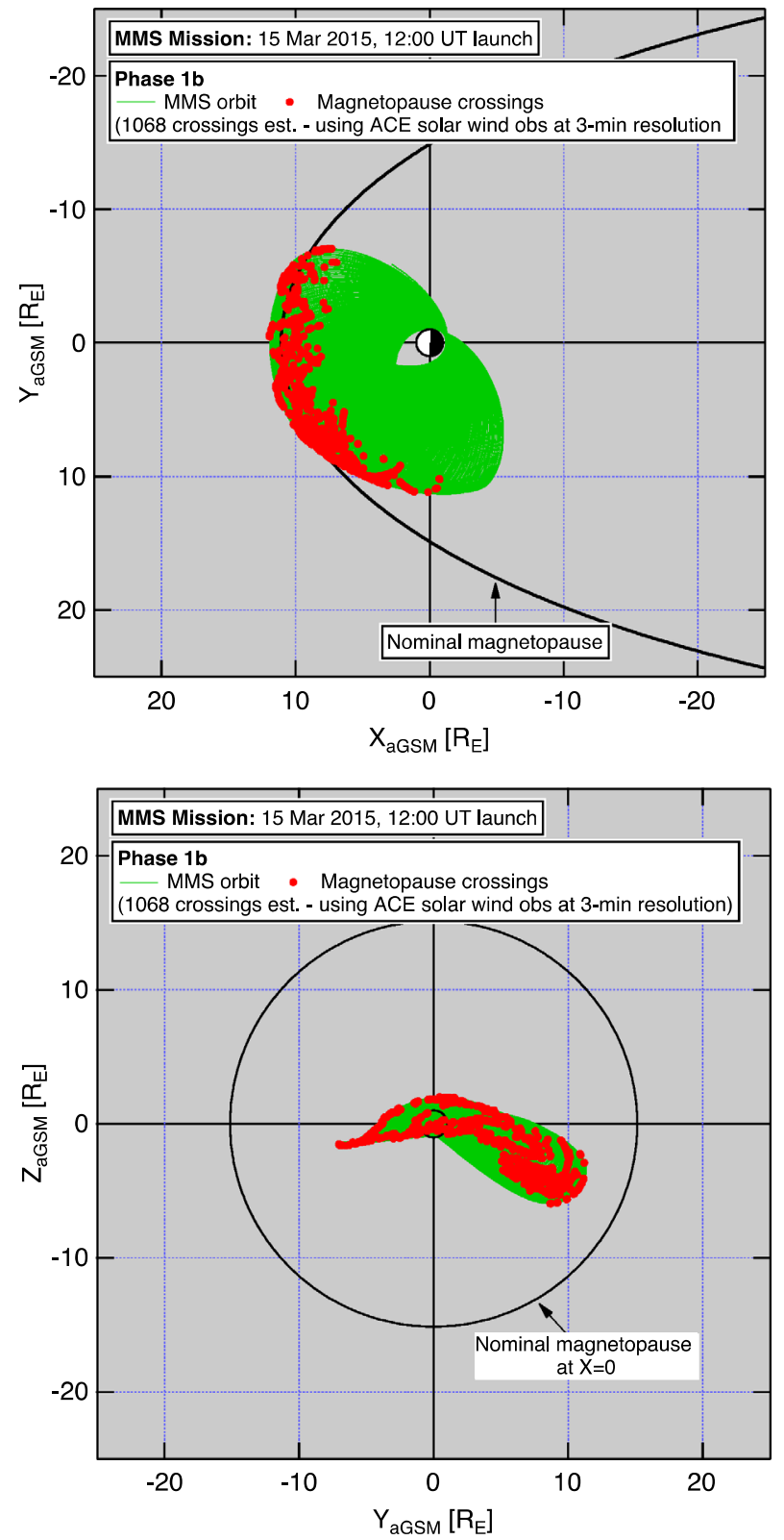

the MMS formation passes inside the location where reconnection is expected to occur most often in the tail and well below the nominal location of the Earth's neutral sheet (see Fig. 5). As a transitional phase, Phase $1 \mathrm{x}$ does not target a reconnection region, and thus specific science objectives have not been defined for it. However, the spacecraft spend a significant amount of time in the high (geomagnetic) latitude lobe regions and may investigate dipolarization fronts that result from reconnection in the magnetotail associated with geomagnetic substorms (e.g., Nakamura et al. 2002, 2012; Runov et al. 2009). 
Fig. 4 Maps of the probability of encountering the electron diffusion region for Phase 1a (top) and $1 \mathrm{~b}$ (bottom) if the spacecraft is at the magnetopause. These maps were produced by projecting the predicted location of the reconnection line onto the $Y-Z$ plane for the entire $\sim 6$-month period when MMS is on the dayside for Phase 1a and $1 \mathrm{~b}$. The predicted MMS magnetopause encounters (determined in the same way as in Figs. 2 and 3) are shown by the plus signs. The MMS magnetopause crossings roughly follow the regions at low latitudes, where the probability of encountering the diffusion region is highest
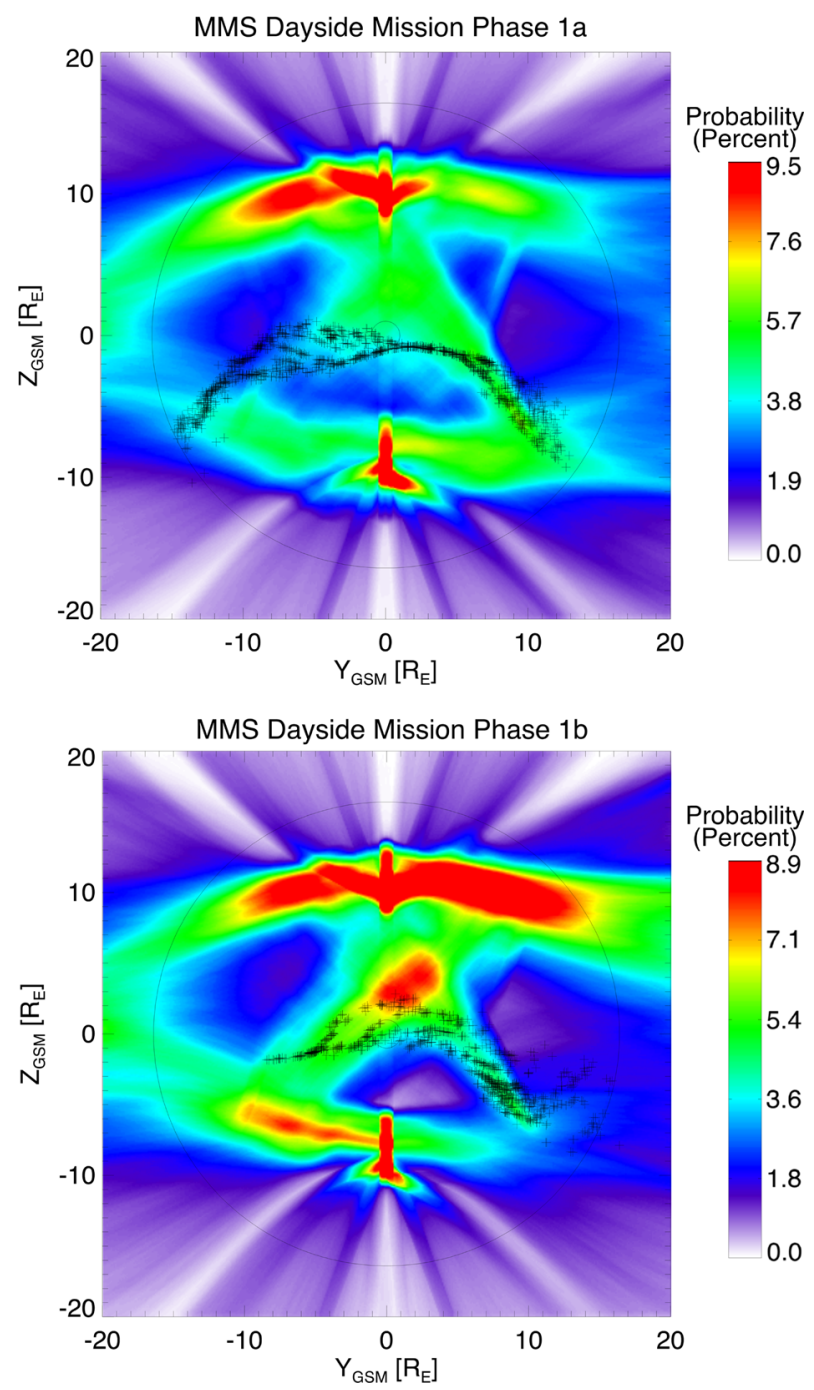

\subsection{Phase 2: Targeting Reconnection in the Magnetotail}

Phase 2 consists of two sub-phases, 2a and 2b (Fig. 6). During Phase 2a, the apogees of the four spacecraft are raised from the Phase 1 apogee of $12 R_{E}$ to $25 R_{E}$ in a series of discrete steps. Although additional magnetopause crossings occur during this phase, the spacecraft may not be in the nominal configuration to study the diffusion region because their apogees may be raised individually or in pairs. Once apogee raising maneuvers are completed and the tetrahedral formation has been re-initialized, Phase $2 b$ and the investigation of the reconnection region in the magnetotail begin.

Unlike predictions of reconnection at the magnetopause, there are no models that use solar wind parameters to predict the location of magnetotail reconnection. Magnetic reconnection in the near-Earth magnetotail occurs between oppositely directed magnetic field lines at the neutral sheet and is associated with episodic, explosive releases of magnetic energy in a 
Fig. 5 MMS Phase 1x orbits viewed from the dusk side of the magnetosphere. The format is the same as in Figs. 2 and 3. The spacecraft are far from the Earth's neutral sheet and earthward of the region where reconnection occurs most frequently in the near-Earth magnetotail. During this phase, the spacecraft will observe depolarization fronts that are the consequence of reconnection in the near tail and substorm development
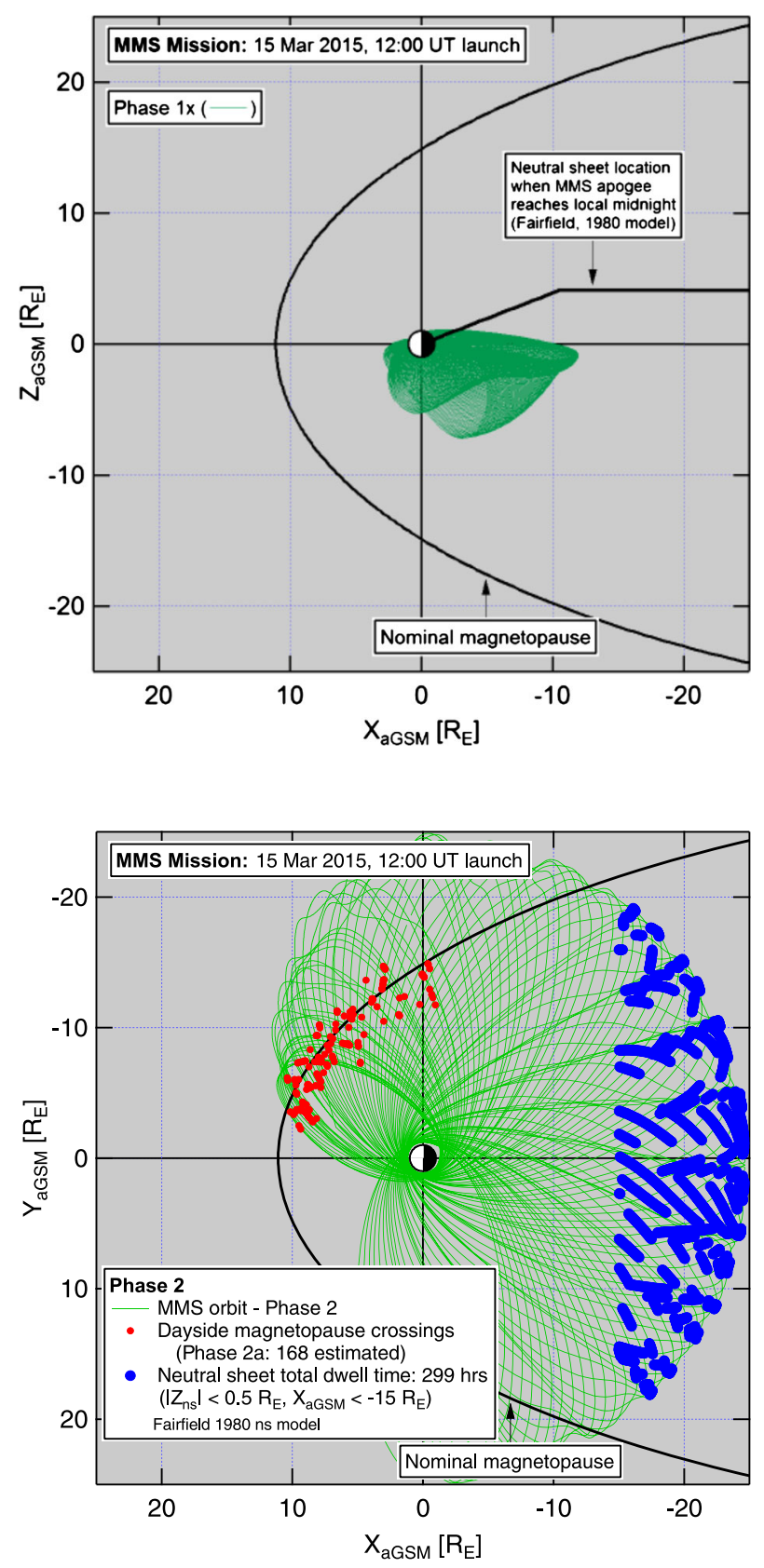

Fig. 6 Phase $2 \mathrm{a}$ and $2 \mathrm{~b}$ orbits projected onto the GSM $X-Y$ plane. Phase 2a begins at 10:00 GSE local time. During this phase, orbit apogees are raised in steps from $12 R_{E}$ to $25 R_{E}$. When the apogee raising maneuvers are complete and the orbit intersects the dawnside magnetopause, Phase $2 \mathrm{~b}$ begins. Phase $2 b$ orbits passes through the magnetotail and the purple dots show times when the spacecraft are within $0.5 R_{E}$ of the magnetotail neutral sheet and $>15 R_{E}$ from the Earth in the $-X$ direction. During these times, there is a significant probability that the spacecraft will encounter the magnetotail reconnection diffusion region

magnetospheric substorm. A near-Earth reconnection line forms across the magnetotail, and plasma is expelled at high speed down the tail. Eventually, this neutral line moves tailward as well. Thus, unlike reconnection at the magnetopause, reconnection in the magnetotail is intermittent and, while it is associated with IMF $B_{z}<0$, there are no predictions for the location in the magnetotail based on the orientation of the IMF. 
Fortunately, there are a few statistical studies of encounters with high-speed tailward plasma flows and encounters with the reconnection region at the near-Earth magnetotail neutral sheet ( $<30 R_{E}$ from the Earth). The studies most relevant to the MMS mission are those that use Geotail data because the Geotail orbit is similar to the MMS Phase 2b orbit. Nagai et al. (2005) showed that high-speed tailward flow events in the Geotail data are very rare when a spacecraft is $|X|<15 R_{E}$ from the Earth. Thus, the near-Earth reconnection line most often forms tailward of this location. Furthermore, they investigated encounters with the reconnection (ion) diffusion region (identified by an encounter with the tail neutral sheet where there is a switch from high-speed tailward flow to high-speed earthward flow and other criteria) and showed that the probability of encountering this region was relatively constant for spacecraft distances $>18 R_{E}$ from the Earth. When properly normalized, the encounters analyzed by Nagai et al. (2005) yield a prediction for the number of reconnection diffusion region encounters for MMS (Genestreti et al. 2013).

Since reconnection region encounters occur when a spacecraft is at the tail neutral sheet, predictions of the number of reconnection diffusion region encounters require a model for the neutral sheet. For the MMS mission design, the Fairfield (1980) empirical neutral sheet model is used. This neutral sheet model is based on a large data set of neutral sheet encounters and has the advantage that the location of the neutral sheet depends only on date and time. For the predicted orbits for the nominal launch date of 15 March 2015 (Fig. 6), there are a total of 299 hours within $0.5 R_{E}$ of the neutral sheet when the spacecraft are $>15 R_{E}$ from Earth in the $X$ direction. As can be seen in Fig. 6, these time periods near the neutral sheet, indicated in the figure by the purple dots, are distributed relatively evenly across the magnetotail.

Genestreti et al. (2013) re-analyzed the reconnection diffusion region encounters originally studied by Nagai et al. (2005) and determined the encounter probability as a function of the number of hours within $0.5 R_{E}$ from the Fairfield model neutral sheet. They found that the probability (per hour near the neutral sheet) is relatively constant at $\sim 3 \%$ for spacecraft distances $|X|>15 R_{E}$ from the Earth. Thus, during the 299 hours that MMS spends within $0.5 R_{E}$ of the model neutral sheet (Fig. 6), the spacecraft are expected to encounter the diffusion region about 11 times.

High-speed tailward flow events occur more frequently. Genestreti et al. (2013) found that the probability (per hour near the neutral sheet) of observing a tailward flow event is relatively constant at $\sim 20 \%$ for spacecraft distances $|X|>15 R_{E}$ from the Earth. Thus, during the 299 hours that MMS spends within $0.5 R_{E}$ of the neutral sheet, the spacecraft are expected to observe about 57 high-speed tailward flow events. Since the reconnection line retreats tailward after its formation (e.g., Eastwood et al. 2010), the MMS spacecraft have a reasonable chance of encountering the reconnection line from these tailward flow events. These would be additional diffusion region encounters over and above the 11 expected encounters. An extension of this study, including the Cluster data set is currently underway.

\section{Mission Profile: Orbit Selection and Design}

As described in the preceding section, the MMS orbit was selected and optimized in an process that involved the iterative development of a design that satisfied both a relatively loose set of science constraints and a more stringent set of engineering constraints and requirements. The final orbit is the result of extensive trade space analysis and modeling. The orbit design process is described in detail by Tooley et al. (2014). 


\subsection{Science and Engineering Constraints}

The constraints imposed by the MMS science objectives on the orbit emerge clearly from the preceding discussion of the magnetospheric regions where the probability of encountering the diffusion region is highest and are summarized in the following paragraphs.

(1) During Phase 1, the focus of the mission is on the dayside magnetopause, the location of which at the subsolar point varies between $9 R_{E}$ and $13 R_{E}$, with an average location of $10 R_{E}$ geocentric. The Phase 1 orbit apogee must maximize magnetopause encounters and has therefore been specified to be $12 R_{E}$. To accommodate variations in the magnetopause location, the nominal science region of interest for Phase 1 is defined as the region $>9 R_{E}$, which ensures that the spacecraft will encounter the magnetopause $>95 \%$ of the time out to $12 R_{E}$. Some adjustment of the science region of interest may be made when the orbit is on the flank of the magnetopause.

(2) Because the probability of encountering the dayside reconnection region is higher near the subsolar point than in surrounding magnetopause regions, the spacecraft must collect data as close to the subsolar point as possible within the mission's engineering and operational constraints. Phase $1 \mathrm{a}$ (Phase $1 \mathrm{~b}$ ) orbit is therefore required to pass within $\pm 20^{\circ}$ $\left( \pm 25^{\circ}\right)$ of the subsolar point (the Earth-Sun line) between 14:00 and 10:00 GSE local time. (The requirement is relaxed somewhat for Phase $1 \mathrm{~b}$ to accommodate the Phase $2 \mathrm{a}$ apogee raise maneuvers, which begin when the apogee reaches 10:00 GSE local time.) Because the orbit is specified to pass within a certain latitude through the subsolar point with no latitude bias above or below, this relatively loose science constraint can be satisfied regardless of the time of year of the launch.

(3) In Phase 2, MMS targets the near-Earth magnetotail, where the probability of encountering the diffusion region and/or a tailward-retreating reconnection line is greatest at downtail distances $>15 R_{E}$. The orbit apogee must therefore be increased from $12 R_{E}$. The science region of interest for Phase $2 \mathrm{~b}$ is specified to be the region tailward of $15 R_{E}$ out to apogee at $25 R_{E}$. The spacecraft are in this region during $\sim 30 \%$ of the orbit. The percent time for the region of interest is constrained by total data storage capacity and the spacecraft telemetry rate.

(4) As discussed above, the probability of encountering the diffusion region in the tail also depends on the amount of time the spacecraft spend within $\pm 0.5 R_{E}$ of the neutral line. To ensure a reasonable chance of encountering the diffusion region, the spacecraft must spend $>100$ hours within $\pm 0.5 R_{E}$ of the Fairfield model neutral sheet at $|X|>15$. (For $<100$ hours, only $1-2$ diffusion region encounters are predicted.)

In addition to the constraints derived from the MMS science objectives, the orbit must satisfy specific engineering requirements regarding (1) the duration and depth of Earth's shadow that the spacecraft can experience without restricting their normal operations and

(2) fuel usage.

(1) During the first two weeks of the mission, when instrument and spacecraft systems are just being turned on and commissioned, the duration of the maximum shadow (umbra $+50 \%$ penumbra) is restricted to $<60$ minutes. Subsequently, for any given 20 -hour period, the maximum shadow duration is restricted to $\leq 231$ minutes duration and the maximum umbra duration to $\leq 216$ minutes.

(2) To ensure that the mission does not run out of fuel, fuel usage is restricted to $<388 \mathrm{~kg}$ of propellant for each spacecraft. This accounts for a $20 \mathrm{~kg}$ allocation for attitude control and for $2 \mathrm{~kg}$ of unusable propellant that will be trapped in the lines. It also includes the 
Fig. 7 Output from SWM76. Differently colored lines show regions of constraint violation. The set union of all allowable regions leads to a daily launch window (launch vehicle RAAN and AOP) shown in the unfilled region in white

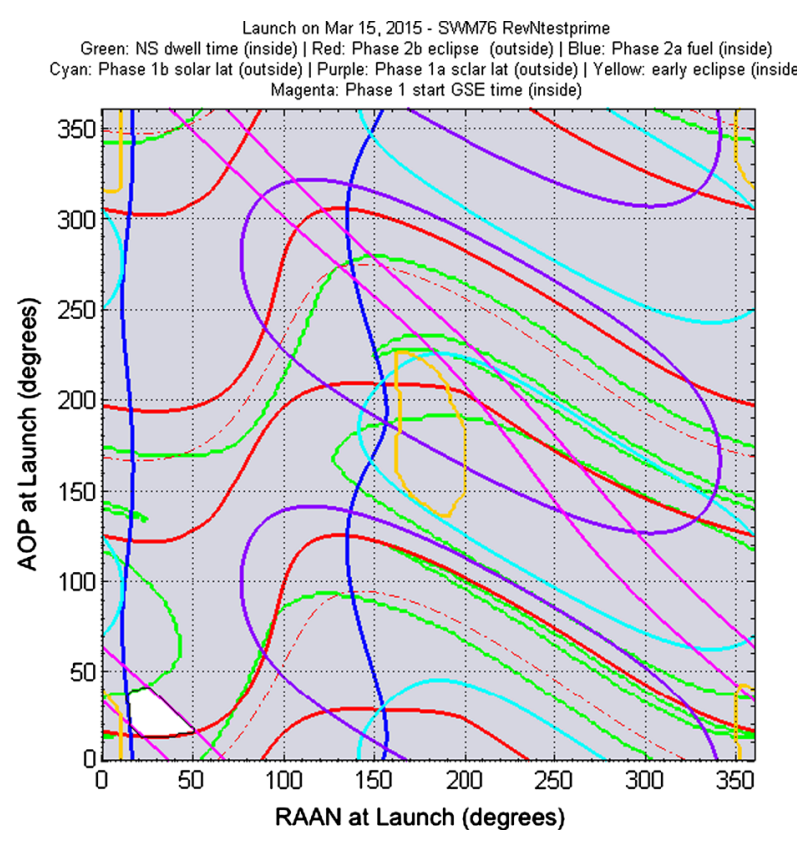

propellant used for deterministic maneuvers (perigee and apogee raising) and stochastic maneuvers (formation maintenance and re-sizing) and the associated knowledge and control errors that arise from uncertainties in the orbit determination and maneuver execution processes, respectively.

A detailed discussion of these and the other engineering requirements and constraints placed on the MMS orbit is given in the orbit design chapter (Tooley et al. 2014).

\subsection{Launch Window Selection}

The MMS launch window is defined as the set of all days and times-of-day in which a launch will allow the constraints on the Phase $1 \mathrm{a} / 1 \mathrm{~b}$ latitude range, Phase $2 \mathrm{~b}$ neutral sheet dwell time, umbra/shadow duration, and fuel usage to be met. For a given injection right ascension of ascending node (RAAN) and argument of perigee (AOP), the Flight Dynamics group used a simple analytic model based on Gauss' planetary equations, SWM76 (Williams 2012), to identify launch window opportunities that are allowed by the above constraints. Results were then verified with high-fidelity simulations. The SWM76 output for the nominal 15 March 2015 launch date is shown in Figs. 7 and 8. Figure 7 show all constraints over the entire range of RAAN versus AOP space. The width in RAAN (for a given AOP) corresponds directly to the duration of a daily launch window, with Earth's rotation rate of 15 degrees/hour acting as the conversion factor. Because of modeling errors and launch vehicle dispersions, a 10-degree width in RAAN is required for a 30-minute launch window. Generically, the allowable regions represent a very small area of the RAAN-AOP space in Fig. 7. However, these allowable regions are still larger than are needed by the MMS launch window, and the resulting freedom can be exploited to maximize science. Figure 8 shows the bounding curves of the science requirements and engineering constraints, the resulting region of allowable RAAN-AOP values, and the Phase 2 neutral sheet dwell times within this region. An AOP value for a representative 30-minute daily launch window is shown. This launch window was chosen because it tends to maximize neutral sheet dwell time. 
Fig. 8 An expanded view of the set of allowable launch states shown in Fig. 8 along with annotations of the values of neutral sheet dwell time (blue), The region of allowable launch states is bounded by the neutral sheet dwell time (green line), the phase $2 \mathrm{~b}$ eclipse (blue line), the phase $2 \mathrm{a}$ fuel (red line), and the phase 1a solar latitude (purple lines). Within this region, the 30-minute launch window chosen for the launch vehicle (grey arrow) tends to maximize neutral sheet dwell time

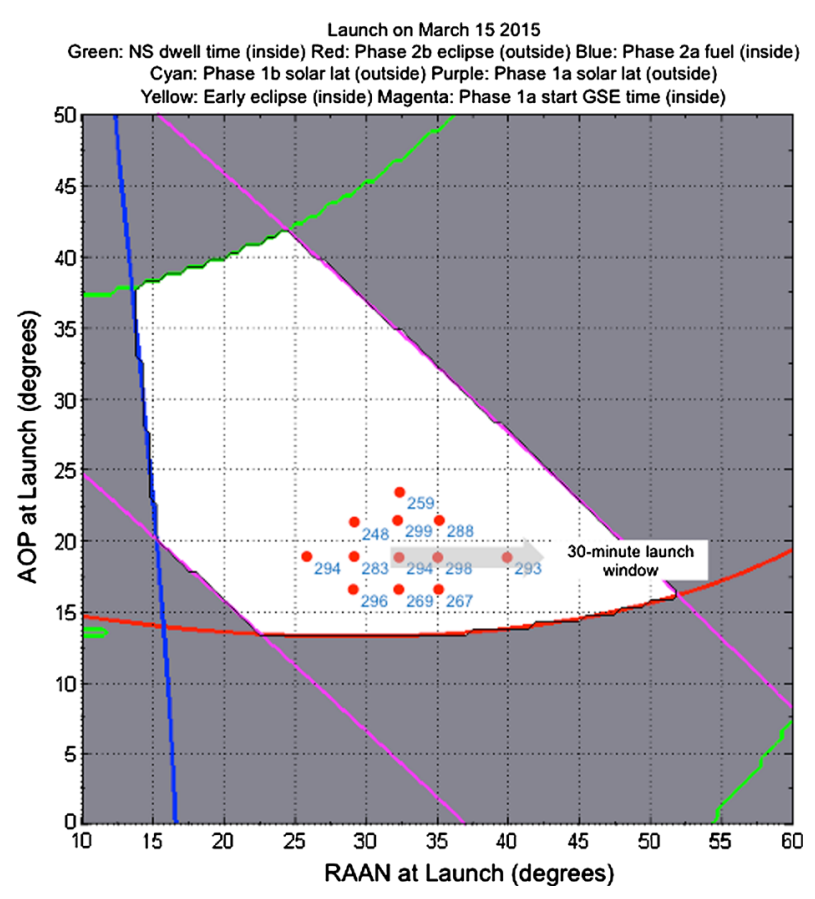

\subsection{Orbit Phases}

As indicated in Sect. 2 above, the two science mission phases comprise five distinct orbital phases, during which the motion of the line of apsides in the slowly rotating GSE reference frame moves the apogee along the dayside magnetopause and through the near-Earth magnetotail. It is this motion of the line of apsides that enables the MMS formation to probe the dayside and nightside reconnection regions. In addition, there is a launch and commissioning phase (Phase 0). The orbital phases are illustrated schematically in Fig. 9 and briefly described in the following paragraphs.

Phase 0: Launch and Commissioning The four MMS spacecraft are launched in a stacked configuration from the Kennedy Spaceflight Center on an Atlas 421 into a low-inclination $\left(28.5^{\circ}\right)$ elliptical orbit with an initial perigee of $1.08 R_{E}$ and an apogee of $12 R_{E}$. The minimum inclination is dictated by the latitude of the launch site, but otherwise it is allowed to float so that optimal performance is obtained. The injection RAAN and AOP are selected from a set of values that vary day-by-day such that the GSE local time of the first apogee is roughly 02:00. From launch plus 4 days $(L+4)$ to $L+14$ days, each spacecraft executes a series of maneuvers to raise perigee from the initial perigee of $1.08 R_{E}$ to $1.2 R_{E}$. After the perigee is raised, the orbit period is $\sim 1$ day. Phase 0 lasts 120 days, during which time instrument and spacecraft systems are commissioned. At approximately $L+96$ days, the spacecraft are placed into a tetrahedral formation with a characteristic scale size of $160 \mathrm{~km}$ to prepare for Phase 1a science operations.

Phase 1a: Magnetopause Reconnection I Science operations start at $L+120$ days when the apogee is within \pm 1 hour $\left( \pm 15^{\circ}\right)$ of the dusk terminator line (i.e., between 19:00 and 17:00 GSE local time). This phase lasts approximately 180 days. The start of science operations within the GSE local time range is used to optimize the encounters with the Earth's 


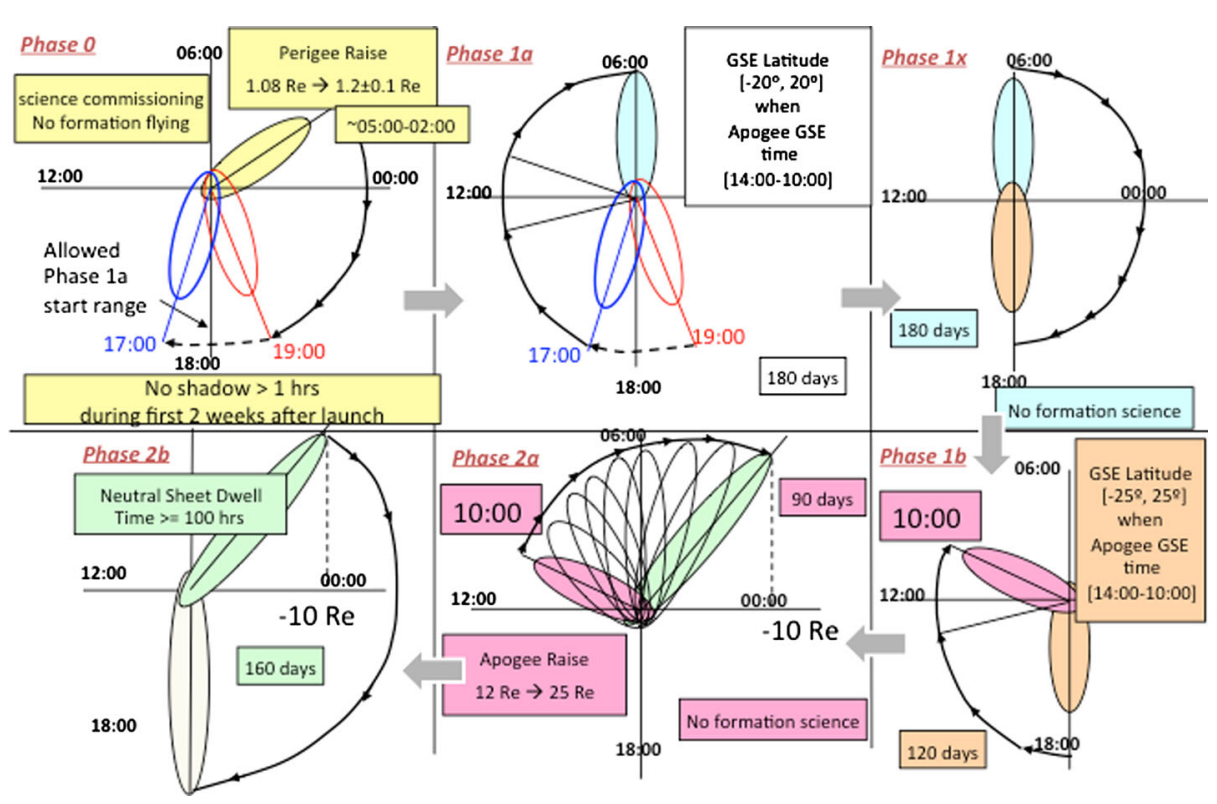

Fig. 9 Visual summary of the formation center's orbital motion through each mission phase as shown in the GSE frame (Earth-Sun line fixed on the horizontal, Sun to the left, ecliptic pole pointing out of the page). The phases are ordered clockwise starting from the top left with Phase 0 and ending on the lower left with Phase $2 b$

magnetotail neutral sheet in Phase 2b. In this sense, this start time is dictated by orbit perturbation from Earth's oblateness. When apogee is between 14:00 and 10:00 GSE local time, the orbit remains within $\pm 20^{\circ}$ of the ecliptic plane, maximizing the probability that the spacecraft formation will encounter the diffusion region (cf. Fig. 2, bottom panel). During the first two months of Phase 1a, the scale size of the formation is adjusted as described below in Sect. 4.

Phase 1x: Transition to Phase $1 b$ The nightside transition phase begins when the apogee reaches a GSE local time of 06:00 and ends at a GSE local time of 18:00 and lasts approximately 180 days. The tetrahedral formation is maintained to optimize fuel use, and science data are collected. However, there are no specific formation science objectives.

Phase 1b: Magnetopause Reconnection II This phase is similar to Phase 1a, but lasts only 120 days. Phase $1 \mathrm{~b}$ ends with apogee at 10:00 GSE local time to allow sufficient time for the apogee raising maneuvers in Phase $2 \mathrm{a}$. As noted above, the latitude constraint is relaxed from $\pm 20^{\circ}$ in Phase 1 a to $\pm 25^{\circ}$ in Phase $1 \mathrm{~b}$.

Phase 2a: Apogee Raising During Phase 2a, the apogee is increased from $12 R_{E}$ to $25 R_{E}$ over approximately 90 days, while the perigee remains unchanged. The orbital period increases from $\sim 1$ day to 2.78 days. No inclination is specified because the orbit inclination is allowed to evolve naturally during the $\sim 1.5$ years after launch. The apogee-raising maneuvers are performed during eight successive perigee passes, with only one spacecraft executing a burn per each pass in the order 1-2-3-4 and then 4-3-2-1. At the end of the apogeeraising campaign, the tetrahedral formation is re-initialized with interspacecraft separations of $400 \mathrm{~km}$. The apogee raising technique is discussed in detail by Tooley et al. (2014). 
Phase 2b: Magnetotail Reconnection The tail phase begins when the apogee reaches a downtail distance of $15 R_{E}$ and ends with the apogee at dusk (18:00 GSE local time). It lasts 160 days. During the initial two months of Phase $2 b$, the formation scale size is adjusted as described in Sect. 4.

Extended Mission and Disposal Mission simulations indicate that the spacecraft will have enough fuel to continue formation flying for 1-2 years after the end of Phase $2 b$, resulting in, for example, at least 100 extra hours of neutral sheet dwell time during an extended mission. After the fuel is depleted, each spacecraft can continue to collect data until re-entry, although the shadows when apogee is tailward become longer than the spacecraft systems can handle without impact to routine operations. The spacecraft will re-enter the Earth's atmosphere approximately 13-16 years after the end of the science mission.

\section{Spacecraft Formation Design}

The four MMS spacecraft are placed into a tetrahedral formation near the end of the commissioning phase and remain in that configuration through Phase 1 . They break formation during Phase 2a, and the tetrahedron is re-established after apogee raising is completed. Periodic formation maneuvers are required during the formation flying phases of the mission owing to the natural degradation of the tetrahedron. Additionally, resizing maneuvers are performed during the first two months of Phase 1 and Phase $2 b$ to determine the optimum formation size for the particular science region of interest.

\subsection{Formation Scale Size}

The scale sizes of the diffusion regions at the dayside magnetopause and in the near-Earth tail are not known at present and are certainly different for the two magnetospheric regions (e.g., Hesse et al. 2014, this issue). Furthermore, the ratio of the thickness to the width of the diffusion region is defined by the reconnection rate, and this rate is poorly determined (see, e.g., Fuselier et al. 2010 and Fuselier and Lewis 2011).

At one extreme, the diffusion region at the magnetopause could be as thin as $1 \mathrm{~km}$ and the width could be $<10 \mathrm{~km}$. At the other extreme, the diffusion region in the magnetotail could be $\sim 30 \mathrm{~km}$ thick and $\sim 300 \mathrm{~km}$ wide. If the diffusion region is as thin as $1 \mathrm{~km}$, all four spacecraft cannot be in the region simultaneously because of engineering constraints placed on the interspacecraft distances. If the diffusion region is as thick as $30 \mathrm{~km}$, then it is possible to have all spacecraft within the region simultaneously if the separations are $<30 \mathrm{~km}$. Fortunately, to achieve the MMS science objectives, the spacecraft can encounter the diffusion region either simultaneously or sequentially. The requirement for a successful or "quality" encounter of the diffusion region is that three of the four spacecraft encounter the diffusion region either simultaneously or sequentially.

Since sequential encounters of the diffusion region are permitted, it is important that the spacecraft separation is of the order of the width of the diffusion region. If the width is as small as $10 \mathrm{~km}$, the spacecraft separation should be also of the order of $10 \mathrm{~km}$ to maximize multiple spacecraft encounters. However, if the diffusion region width is $300 \mathrm{~km}$, then the overall probability of encountering the diffusion region is reduced if the spacecraft separation is significantly smaller than this width. Therefore, the best strategy is to have a flexible plan for spacecraft separations that uses experience from previous diffusion region encounters to determine future separations. 
Table 2 Separation constants and spacecraft separations used to determine the quality factor for the MMS mission

\begin{tabular}{lrrrrrrrr}
\hline & \multicolumn{7}{c}{ Spacecraft separations $(\mathrm{km})$} \\
\cline { 3 - 8 } & & 10 & 25 & 30 & 40 & 60 & 160 & 400 \\
\hline Separation & $L 1$ & 4 & 15 & 19.313 & 25 & 45 & 135 & 250 \\
Constants & $L 2$ & 6 & 20 & 23.15 & 30 & 50 & 140 & 300 \\
& $L 3$ & 18 & 35 & 42.075 & 55 & 75 & 190 & 550 \\
& $L 4$ & 24 & 40 & 49.475 & 65 & 80 & 210 & 600 \\
\hline
\end{tabular}

Since the actual width of the diffusion region is not known, in each science phase a number of spacecraft separations are used to find the optimal separation. The spacecraft separations are used for the entire mission are shown in the second row in Table 2. Since the reconnection diffusion region at the magnetopause is expected to be smaller than that in the magnetotail (Hesse et al. 2014, this issue), the initial and final spacecraft separations are smaller in Phase 1a than in Phase 2b, ranging from $160 \mathrm{~km}$ to $10 \mathrm{~km}$ for Phase 1a and from $400 \mathrm{~km}$ to $30 \mathrm{~km}$ for Phase 2b. During the first two months of each phase, the separations are changed every 15 days. At the end of this process, the science team analyzes the data and determines by consensus the final median scale size for the remainder of Phase 1a and all of Phases 1x and 1b. For mission resource planning, the consensus median scale size has been specified as $30 \mathrm{~km}$ for Phase 1 and $50 \mathrm{~km}$ for Phase $2 \mathrm{~b}$.

\subsection{Formation Quality Factor}

The optimum configuration for the MMS formation is a configuration as close to a regular tetrahedron as possible with separations appropriate to the science region of interest. To describe the quality of the formation, a quantifiable quality factor $(Q)$ is used, an approach successfully employed in Cluster spacecraft operations. For spacecraft safety, the quality factor is used as one measure of spacecraft separation. The predicted spacecraft separations are closely monitored and the MMS flight dynamics team specifies minimum separations. If spacecraft separations are predicted to be lower than these minimums, then a collision avoidance maneuver is executed.

The Cluster mission defined the tetrahedron quality factor in terms of the ratio of the volume of the actual tetrahedron divided by the volume of a regular tetrahedron with sides equal to the average spacecraft separations of the actual tetrahedron. The ratio approaches zero as the four spacecraft approach a single plane. For the MMS mission, spacecraft separation is also an important quantity. Therefore, in addition to the quality of the tetrahedron volume, the relative spacecraft separations are also included in the overall quality factor. The overall quality factor is defined as:

$$
Q=Q_{v} \cdot Q_{s}
$$

where $Q_{v}$ is the quality factor for the tetrahedron shape and $Q_{s}$ is the quality factor for the spacecraft separation. $Q_{v}$ is defined as:

$$
Q_{v}=V_{a} / V_{r}
$$

where $V_{a}$ is the actual volume of the tetrahedron at a given point in the orbit and $V_{r}$ is the volume of a regular tetrahedron of side $L$, the average of the sides of the actual tetrahedron. 
The quality factor for the spacecraft separation is defined through a set of conditions:

$$
\begin{aligned}
& \text { If } L<L 1, \text { then } Q_{s}=0, \quad \text { if } L 2<L<L 3, \text { then } Q_{s}=1, \quad \text { and } \\
& \text { if } L>L 4, \text { then } Q_{s}=0 \\
& \text { If } L 1<L<L 2, \text { then } Q_{s}=(L-L 1)^{2}(L+L 1-2 L 2)^{2} /(L 2-L 1)^{4} \\
& \text { If } L 3<L<L 4, \quad \text { then } Q_{s}-(L-L 4)^{2}(L-2 L 3+L 4)^{2} /(L 2-L 1)^{4}
\end{aligned}
$$

The values for $L 1, L 2, L 3$, and $L 4$ (see Table 2) depend on the desired spacecraft separations, which change during the mission. Overall, Eqs. (3) through (5) provide for a relatively graceful degradation in the quality factor as the spacecraft separations change. The degradation is more graceful at larger separations than at smaller ones. At the smallest separations, the more rapid degradation results in a tetrahedron quality maneuver before a maneuver for collision avoidance is required. Also, the relatively generous allowance for $L 1$ through $L 4$ ranges in Table 2 makes the tetrahedron shape the more important parameter in the overall quality factor in Eq. (1).

\subsection{Evolution of the Formation Quality Factor}

The goodness of a formation is determined by the time evolution, $T_{Q}$, of the quality factor $Q(t)$ in Eq. (1). In Eq. (1), $Q(t)$ has an instantaneous range [0,1] and is a product of two functions as shown in Eq. (1): $Q_{s}(t)$, which limits the interspacecraft spacing in a relatively broad range around the desired value, and $Q_{v}(t)$, which favors formations that are closer to an ideal tetrahedron. A good formation achieves values near $Q(t)=1$ at apogee (close to an ideal tetrahedron with the desired size) and values near zero at perigee (scale size too large) or along the inbound or outbound flanks (contained volume too planar). The definition of $T_{Q}$ is the percentage of time that $Q(t)>0.7$ when the formation is in the region of interest (radius $>9 R_{E}$ in Phase $1 \& R_{E}>15$ in Phase $2 \mathrm{~b}$ ). There is a family of formation initial conditions that give essentially the same value of $T_{Q}$ but which cost different amounts of fuel to establish. A delta-V minimization technique (minFuel) is used to target formation resizing or maintenance maneuvers (Figs. 10 and 11) that optimize the fuel usage subject to achieving a formation that yields a $T_{Q}$ of at least 80 . That is, the MMS spacecraft will be maintained in a tetrahedron configuration with $Q>0.7$ for $80 \%$ of the time when the spacecraft are in the science region of interest.

The constraints on the quality factor in Eq. (1) are the result of a balance between the desire to have an optimum spacecraft configuration and separation and the need to perform orbit maneuvers to maintain the configuration. Keeping a relaxed quality factor and requiring it over only a portion ( $80 \%$ ) of the entire science region of interest reduces the required number of orbit maneuvers. Based on simulations and direct experience with the Cluster mission, these constraints reduce the number of maneuvers for configuration maintenance to approximately one every two weeks. Minimizing the number of maneuvers not only optimizes fuel usage, as discussed above, but also reduces interruption of science operations, if the maneuvers have to occur in the science region of interest.

\subsection{Formation Maneuvers and Close Approach}

Practical considerations limit what can be achieved in operations compared to the computer simulations used in targeting formation maneuvers (Schiff and Dove 2011). Primary among these are the effects that knowledge and control errors (due to orbit determination \& propagation and maneuver execution) have on $T_{Q}$. Onboard orbit determination is via a weaksignal GPS receiver pumping pseudo-range data to an extended Kalman filter. It produces a 


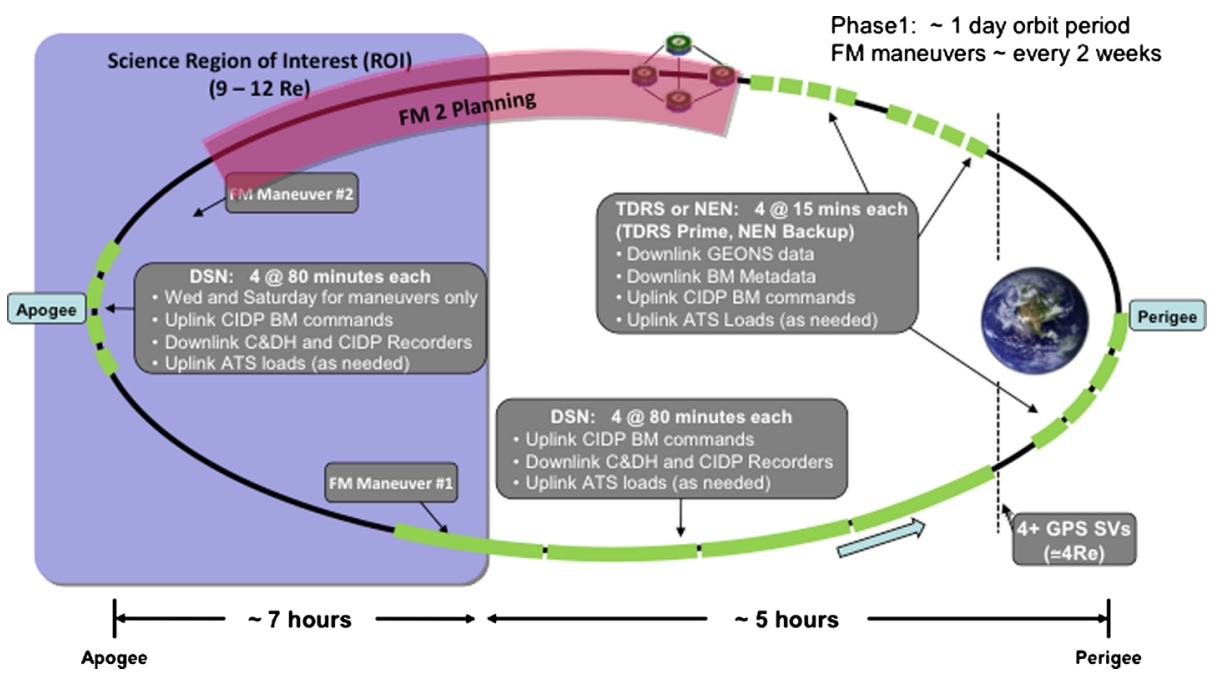

Fig. 10 Orbit operations for Phase 1a and 1b. The purple shading shows the science region of interest, where the instruments are operated in fast survey and obtain both fast survey and burst data. Green boxes on the orbit labeled formation maintenance (FM) maneuvers show times for possible orbit maneuvers. An FM maneuver is performed as a pair of maneuvers with the first occurring just as the formation leaves the region of interest and the second occurring at the subsequent apogee. In general, these occur approximately once every two weeks. Also shown are a set of contacts near perigee used in replanning of the second maneuver based on what happened on the first

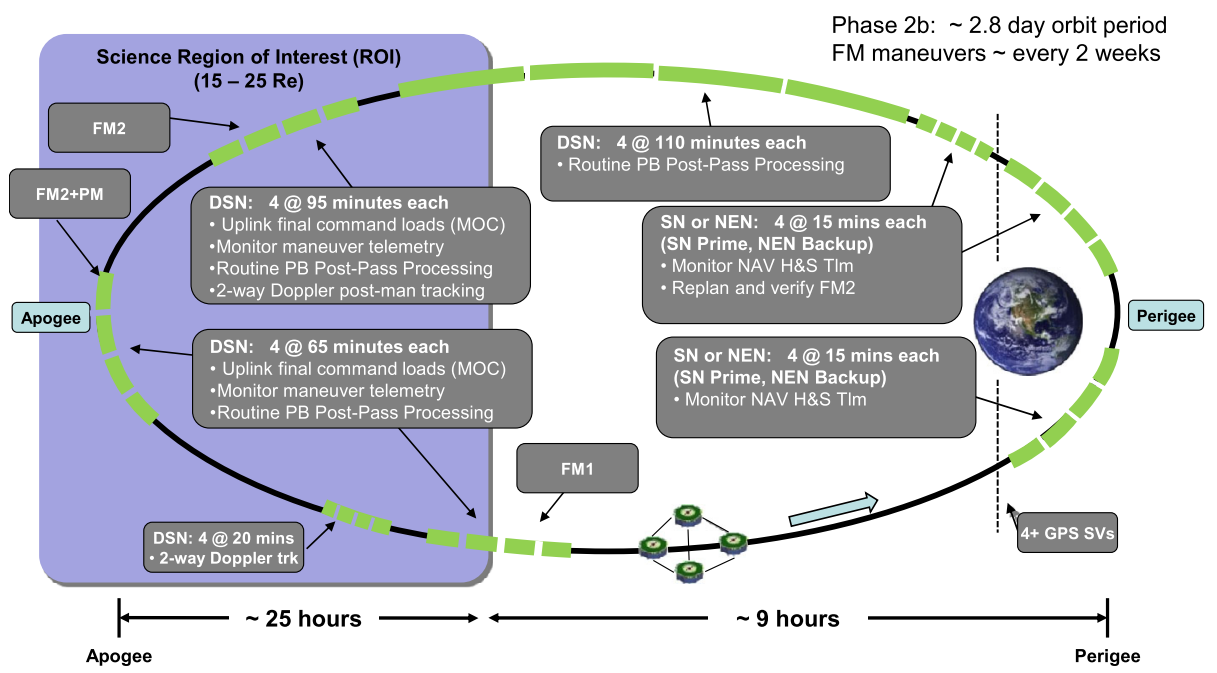

Fig. 11 Orbit operations for Phase 2b. The format is the same as that for Fig. 10

best-estimated state used in maneuver planning that is accurate only to a given level. This state is numerically propagated to the time of the first formation maneuver (FM1) and given to minFuel for targeting. Even if performed exactly, the maneuver will result in subsequent motion near, but not exactly on, the desired trajectory (due to both the uncertainty in the initial conditions and errors in numerical propagation). Significantly larger error (2-10 times) 
Fig. 12 Typical evolution of $T_{Q}$ per orbit in Phase 1 relative to the required threshold value of 80 . Due to knowledge (orbit determination) and control (maneuver execution) errors, it is impossible to achieve a $T_{Q}$ for every orbit over 80 but it is possible to achieve an average value in excess of 91

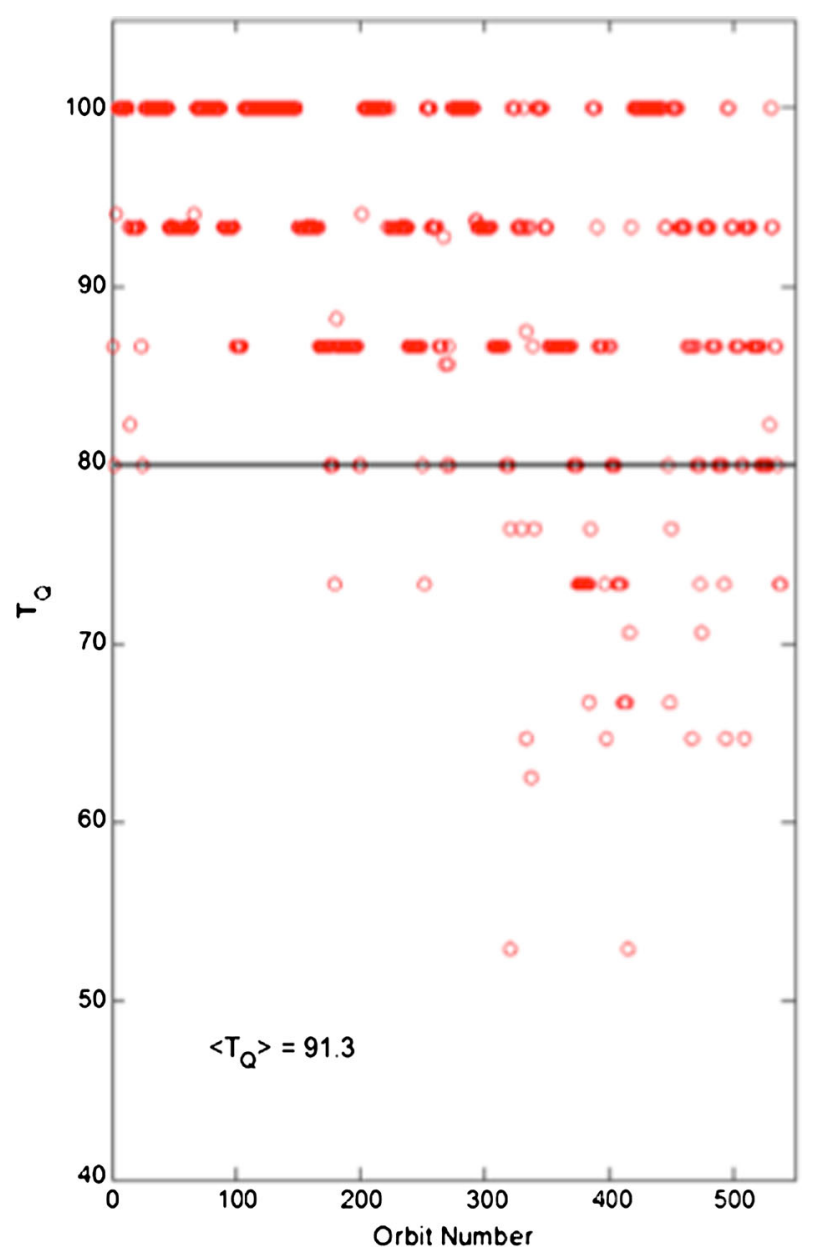

is also introduced by the onboard propulsion system and maneuver controller. The second maneuver of the formation pair is retargeted based on a new estimated state just after the perigee between FM1 and FM2 in order to minimize this error. The overall result of the interplay between the knowledge and control errors is that: (1) it is impossible to guarantee that every non-maneuver orbit will have a $T_{Q}$ greater than 80 (see Fig. 12), although an average of $T_{Q}$ that meets this requirement is easily achieved; and (2) it is possible (although rare) that the realization of these errors will cause an unsafe close approach between at least two of the MMS spacecraft. Assessment and mitigation of unsafe close approaches is a subtle subject. Wawrzyniak et al. (2013) provides details on the two-level hypothesis testing employed to meet the appropriate balance between having a low miss detection rate without having too high of a false alarm rate and overuse of an unplanned maneuver to increase spacing between conjuncting spacecraft.

\section{Mission Science Operations}

There are two basic instrument science operation modes, slow survey and fast survey (cf. Figs. 10 and 11). However, there are three data acquisition rates (slow, fast and burst). The 
operation times are limited by the total data storage and the telemetry rates for the mission. Outside the regions of interest, slow survey mode is used exclusively. The time resolution for the slow survey mode is relatively low to conserve data storage space for data from the region of interest. Within the region of interest, the instruments are operated in fast survey mode and data are acquired at both fast survey and burst rates. All data acquired at both fast survey and burst rates in the region of interest are stored onboard at the highest time resolution, and all fast survey data from the region of interest are transmitted to ground. In addition, burst data triggers (see Sect. 5.1) are transmitted to ground. These triggers are used with the scientist in the loop (SITL) to determine which burst data intervals to bring to ground. Only a very small part of the total burst data acquired in the science region of interest can be transmitted to ground. For example, in Phase 1a and 1b, approximately 15 minutes of burst data are available for transmission to ground. While this appears to be very limited, it is sufficient, for example, to capture all of the magnetopause crossings during a single orbit (with an average of 6 magnetopause crossings per orbit, each lasting approximately one to two minutes, accounting for the fact that the spacecraft may not cross at the same time). Since the reconnection diffusion region will be embedded in the magnetopause, the burst data intervals that are transmitted to ground are sufficient to complete the science objectives of the mission.

As illustrated in Figs. 10 and 11, formation maintenance (FM) maneuvers are usually conducted outside the regions of interest. However, there may be times when an FM maneuver is required near or within the science region of interest. Therefore, as illustrated in Figs. 10 and 11, the operations team reserves some time at apogee and near the end of the science region of interest for these maneuvers. They will be used only if necessary because they require taking some instruments out of science operation mode to perform the maneuver, and this reduces the science data obtained in the critical science region.

While key data are stored onboard for the entire science region of interest, some prioritization of the data transmitted to ground is needed. This prioritization is particularly important during the magnetotail phase (Phase 2b), when the longer orbit period limits the number of burst data intervals that can be transmitted to ground. Furthermore, since MMS is a multispacecraft mission, this prioritization must include selection of the best events from a multi-spacecraft perspective. The burst data "trigger" system has the task of prioritizing the onboard data. For the MMS mission, this burst data trigger system is supported on the ground by a scientist-in-the-loop.

\subsection{Burst Data Selection and the Scientist in the Loop}

The "slow survey" and "fast survey" data are designed to be as uninterrupted as possible throughout the MMS mission. These data have time resolutions comparable with, and in many cases faster than that of previous magnetospheric missions. The "burst data," detailed in the instrument descriptions (Mauk et al. 2014, this issue; Pollock et al. 2014, this issue; Torbert et al. 2014, this issue; Young et al. 2014, this issue), have time resolutions (and thus spatial resolutions) that are one to two orders of magnitude higher. These data contain valuable measurements of the fields and plasma in the electron and ion diffusion regions at the cadence required to achieve the science objectives. In addition, these data are valuable for investigations of acceleration and turbulence.

The burst data volume, however, far exceeds the capabilities (bandwidth) of the MMS telemetry transmission system. Even though roughly $75 \%$ of the telemetry bandwidth is allocated to burst data, only $\sim 2 \%$ of burst data (approximately 15 minutes per day) can be transmitted due to bandwidth constraints. The MMS burst system is designed to select the most scientifically valuable segments of these data. 
The basic strategy of MMS mission is to store all of the burst data obtained during an orbit ( $\sim 1$ to 3 days) in mass memory and then later select the most scientifically interesting segments of burst data (15 minutes for a 1-day orbit up to 45 minutes for a 3-day orbit) for transmission. The selection of the scientifically interesting periods is based on data quality values calculated on board (see below) and, if possible, on survey data.

The burst data intervals are selected in one of two ways. An automatic selection is made based on data quality values (see below), which are a calculated on board each of the spacecraft and transmitted several times an orbit. Alternatively, a scientist makes the selection of the burst data based on viewing the survey data and data quality values. The latter method is called Scientist-In-The-Loop (SITL).

Mass Memory, Trigger Data, and Cycle Data Quality (CDQ) The MMS mass memory in the Central Instrument Data Processor (CIDP) is described in detail elsewhere (Tooley et al. 2014 , this issue). Here, only a brief review of the important facts is provided. The majority of the 96 gigabyte (GB) mass memory (flash-based RAM) is divided into $4 \times 2^{20}$ byte (called $4 \mathrm{M}_{2}$ Byte) "burst data buffers". The mass memory has up to $\sim 24,000$ of the 4 megabyte (MB) buffers. Each burst buffer stores $10 \mathrm{~s}$ of burst data from all instruments, allowing for up to $\sim 50$ hours of burst data storage (some of the mass memory must be used for survey data). Thus, all of the burst data in the science region of interest (roughly half of the time in a given orbit) is stored on board.

The burst data volumes varies due to compression efficiency or instrument configuration, so the entire $4 \mathrm{M}_{2}$ Bytes of physical storage may not be used during a $10 \mathrm{~s}$ interval. The burst buffers are assigned a start time on $10 \mathrm{~s}$ boundaries of the spacecraft clocks, which are synchronized through GPS reception. The start (and stop) times of the data packets from the instruments are not necessarily on these $10 \mathrm{~s}$ boundaries. If the data packet from an instrument starts within the $10 \mathrm{~s}$ burst buffer period, the entire packet is stored in that buffer. There are expected to be small ( $\ll 1 \mathrm{~s}$ for a majority of the data) delays in the start times of differing types of data within a $10 \mathrm{~s}$ buffer. No burst data are lost in this process. Each data packet is stored in a $10 \mathrm{~s}$ burst buffer, depending on its start time.

Each $10 \mathrm{~s}$ burst buffer on each of the spacecraft is assigned a "Cycle Data Quality" (CDQ) value, an integer number between 0 and 255. This number is calculated by a linear combination of 34 onboard trigger data values (Table 3 ) calculated by the instruments and supplied to the CIDP:

$$
C D Q=\frac{\sum w_{i}\left(T D_{i}-O S_{i}\right)}{\sum w_{i}},
$$

where $T D_{i}$ is the trigger data value provided by the instruments, $O S_{i}$ is an offset, and $w_{i}$ is a weighting factor. The spacecraft transmit CDQ values several times during an orbit.

The weighting factors $\left(w_{i}\right)$ and offsets $\left(O S_{i}\right)$ are stored in tables held on board each of the spacecraft in the CIDP. These factors and offsets are reconfigurable. Initially, the weighting factors are configured such that the CDQ increases with large changes in plasma density and reversals of the magnetic field (i.e., when the magnetopause current layer is crossed). New weighting factors and offsets will be developed for later in the mission for crossings of the Earth's neutral sheet (where there is a field reversal, but not necessarily a large change in plasma density).

Automatic Selection Automatic selection is the default process for burst data prioritization. This selection uses the array of CDQ values from the four spacecraft (Fig. 13). These values have a $10 \mathrm{~s}$ cadence corresponding to the $10 \mathrm{~s}$ of data in each of the $4 \mathrm{M}_{2}$ Byte burst data 
Table 3 Trigger data values supplied by the MMS instruments. The data values are reprogrammable to optimize the priority selection of the highest quality reconnection events

\begin{tabular}{|c|c|c|c|}
\hline ID & Instrument & Measurement & Description \\
\hline 0 & FPI & Electrons & Pseudo-content variance \\
\hline 1 & FPI & Electrons & Pseudo-content Idelta of meanl \\
\hline 2 & FPI & Electrons & Parallel Pseudo-flux variance \\
\hline 3 & FPI & Electrons & Parallel Pseudo-flux mean \\
\hline 4 & FPI & Electrons & Directional Pseudo-content variance \\
\hline 5 & FPI & Electrons & Directional Pseudo-content mean \\
\hline 6 & FPI & Ions & Pseudo-content variance \\
\hline 7 & FPI & Ions & Pseudo-content Idelta of meanl \\
\hline 8 & FPI & Ions & Pseudo-flux variance in $\mathrm{S} / \mathrm{C} Z$ direction \\
\hline 9 & FPI & Ions & Pseudo-flux variance in S/C $X$ direction \\
\hline 10 & FPI & Ions & Pseudo-flux variance in S/C $Y$ direction \\
\hline 16 & Fields & $E_{\|}$ & Average of 4 highest $\left|E_{\|}\right|$in $10 \mathrm{~s}$ \\
\hline 17 & Fields & E & Average of 4 highest $|E|$ in $10 \mathrm{~s}$ \\
\hline 18 & Fields & $\delta B_{\mathrm{Z}}$ & Average of 4 highest $\left|\delta B_{z}\right|$ in $10 \mathrm{~s}$ \\
\hline 19 & Fields & $E_{R M S 1}$ & Approximate RMS of $E, 10 \mathrm{~Hz}-100 \mathrm{~Hz}$ \\
\hline 20 & Fields & $E_{R M S 2}$ & Approximate RMS of $E, 100 \mathrm{~Hz}-1 \mathrm{kHz}$ \\
\hline 21 & Fields & $E_{R M S 3}$ & Approximate RMS of $E, 1 \mathrm{kHz}-10 \mathrm{kHz}$ \\
\hline 22 & Fields & $E_{R M S 4}$ & Approximate RMS of $E, 10 \mathrm{kHz}-100 \mathrm{kHz}$ \\
\hline 23 & Fields EDI & Electron Flux & Electron Flux at $500 \mathrm{eV}$ or $1 \mathrm{keV}$ \\
\hline 24 & Fields & $\mathrm{EH}$ & Electron hole count in $10 \mathrm{~s}$ \\
\hline 25 & Fields & $\delta B$ & Average of 4 highest $|\delta B|$ in $10 \mathrm{~s}$ \\
\hline 26 & Fields & $1 /|B|$ & Average of 4 highest $1 /|B|$ in $10 \mathrm{~s}$ \\
\hline 27 & Fields & $B_{R M S 1}$ & Approximate RMS of $B, 10 \mathrm{~Hz}-100 \mathrm{~Hz}$ \\
\hline 28 & Fields & $B_{R M S 2}$ & Approximate RMS of $B, 100 \mathrm{~Hz}-1 \mathrm{kHz}$ \\
\hline 29 & Fields & $B_{R M S 3}$ & Approximate RMS of $B, 1 \mathrm{kHz}-10 \mathrm{kHz}$ \\
\hline 30 & Fields & $n$ & Estimate of $n$ from spacecraft potential \\
\hline 30 & Fields & $\delta n$ & Estimate of $\delta n$ RMS from S/C potential \\
\hline 36 & HPCA & Ions & $\mathrm{H}^{+}$energy flux \\
\hline 37 & HPCA & Ions & $\mathrm{O}^{+}$energy flux \\
\hline 40 & EPD & Electrons & High energy electron intensity \\
\hline 41 & EPD & Electrons & High energy electron variability \\
\hline 42 & EPD & Electrons & High energy electron anisotropy \\
\hline 52 & EPD & Ions & High energy electron intensity \\
\hline 53 & EPD & Ions & High energy electron anisotropy \\
\hline
\end{tabular}

buffers. The time arrays of the CDQ values from the four spacecraft are combined to a single time array, called the "Mission Data Quality" (MDQ), by an amplitude-ordered weighting:

$$
M D Q(t)=\sum_{S C} C D Q_{i}(t) \cdot O W_{i}(t)
$$




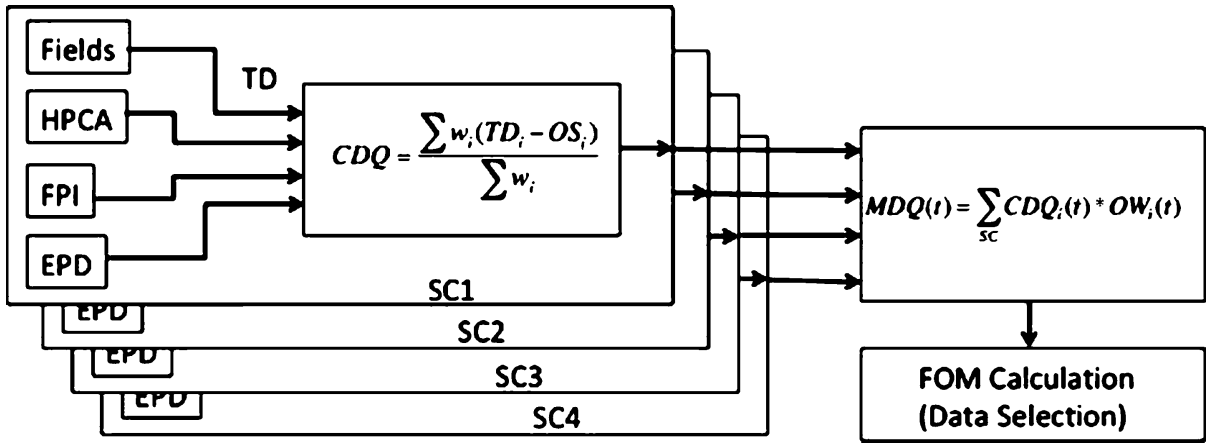

Fig. 13 A schematic of the automatic data selection process. Trigger data (10 s cadence) from each of the instruments on a spacecraft are combined to form "Cycle Data Quality" (CDQ) arrays. CDQ arrays from all of the spacecraft are combined to a single value called the "Mission Data Quality" (MDQ) array with $10 \mathrm{~s}$ cadences. MDQ arrays are used to select burst data segments

where $O W_{i}(t)$ is the amplitude-ordered weighting and the summation is over the four spacecraft. Initially, $O W=[0.4,0.3,0.2,0.1]$. For each time period, the largest CDQ among the four spacecraft is weighted at 0.4 , the second largest is weighted at 0.3 , and so on. The time array of MDQ values reflects the data quality of the four spacecraft combined, with the heaviest weighting on the highest quality value among the spacecraft.

After the MDQ time array is calculated, the most scientifically valuable burst data are selected for download based on the MDQ array. An important element in this selection is to have continuous stretches of data. These continuous stretches of data called "segments", which are a set of $N$ contiguous burst data buffers. The ultimate selection of the most scientifically valuable burst data involves an optimization MDQ values and segment lengths. Each segment is assigned a "Figure of Merit" (FOM), which is an indication of its scientific value (Fig. 13).

Scientist in the Loop (SITL) The automatic process is ultimately based on trigger data, which represent the quality of data in each of the MMS instruments. Along side the automated process, a second selection process may take place. This manual or SITL selection process allows for burst data interval selection by a scientist. The SITL has at their disposal the survey data from MMS, data from other satellites, and ground-based data such as magnetometer chains. This manual selection processes can override the automatic process and allows for a much more informative selection of burst data.

Multiple Orbit Data Collection The burst data selection system allows for hold over of data from previous orbits. Past space missions in the magnetosphere indicate that many reconnection events may occur in a single day, whereas other days may have few such events. While the average burst data collection is limited to roughly 15 minutes of burst data per day, the MMS burst system allows for storage of a large quantity of data selected by the automatic routine or the SITL scientists. This long-term storage of selected data allows for better optimization of the number of reconnection events and transmission to ground of these events in a pre-determined priority order over the period of several orbits.

In summary, the MMS mission features burst data that have cadences that can resolve the electron diffusion region in magnetic reconnection. The burst data volume, however, far exceeds the bandwidth of the MMS telemetry transmission system. The MMS mission is designed to store on board several orbits of data in the science regions or interest. A small 
fraction of the stored data with the highest scientific quality, that is, the highest likelihood of being associated with magnetic reconnection is selected for transmission. The data selection process is automated using on-board "trigger data" supplied by the instruments, which indicate a higher probability of reconnection events. In addition, a manual SITL selection process allows scientists to use other data not available to the automated process to select time periods of the high-cadence burst data that are likely to be associated with magnetic reconnection.

\section{Summary}

The MMS mission is fundamentally different from other magnetospheric missions in that it targets the very small electron diffusion region at the magnetopause and in the near-Earth magnetotail. The targeting is accomplished through a three-step iterative process. First, relatively loose science constraints are placed on the mission design. Second, candidate orbits that meet these science constraints and also the engineering constraints discussed in Sect. 3 are identified. Third, these candidate orbits are analyzed to determine which orbits return the greatest amount of science. These orbits are further refined through this iterative process.

An empirical model for the location of reconnection at the magnetopause indicates that the probability of encountering the reconnection diffusion region maximizes at low latitudes near the subsolar region. Therefore, the MMS orbits are designed to pass through this region during Phases 1a and $1 \mathrm{~b}$ of the mission. Proper choice of the launch local time permits this targeting of the subsolar region and, at the same time, allows a natural evolution of the orbit to maximize the encounters with the neutral sheet in the magnetotail during the second phase of the mission. For the nominal launch date (15 March 2015), a total of 56 encounters with the diffusion region at the magnetopause and approximately 11 encounters with the diffusion region in the magnetotail are predicted. These predictions exceed the 16 quality encounters with the diffusion region on the dayside and nightside that are required for full mission success (no breakdown of the number of encounters on the two sides is specified in these requirements).

The spacecraft will be in a tetrahedron configuration in the science regions of interest (i.e., at the magnetopause and in the magnetotail $>15 R_{E}$ from the Earth). A quality factor (Eq. (1)) is specified for these regions of interest and the efficacy of this quality factor is specified in the mission requirements $(Q>0.7$ for $80 \%$ of the science region of interest). Previous experience with the Cluster spacecraft tetrahedron configuration provides confidence that the quality factor can be maintained as specified over the science regions of interest without excessive orbit maneuvers. Unlike Cluster, the MMS spacecraft separations (see Table 2) will be changed over relatively short periods of time to determine the optimum separation for encountering the electron diffusion regions. Since the size of the diffusion region is not known, four different spacecraft separations are used to determine which spacecraft separation maximizes the probability that at least three of the spacecraft encounter the diffusion region either sequentially or simultaneously. For Phase 1a, and 2b, the four spacecraft separations are changed every 15 days from start of the phase. Once the optimum separation is known, it is used for the remainder of the phase and, in the case of magnetopause reconnection, during the entirety of Phase $1 \mathrm{~b}$.

Outside the science regions of interest, the instrument suite is operated in slow survey mode. Inside the science regions of interest, the spacecraft are operated in fast survey mode and both fast survey and burst data are accumulated and stored on board. All fast survey data are transmitted to ground; however, only a fraction (for example, about 15 minutes 
of data from a Phase 1 orbit) of these burst data can be transmitted to ground. Therefore, the instrument suite has several burst mode triggers that select the highest quality events for transmission to ground. These selections can be overridden on the ground by the scientist-inthe-loop. Although this data management scheme appears to be severely limiting, experience with the THEMIS mission, for example, demonstrates that all magnetopause crossings can be transmitted to ground for Phase 1a and 1b (Phan et al. 2014, this issue). Furthermore, proper burst data quality factors will be able to select the highest priority diffusion region encounters from the magnetotail.

The combination of selective targeting of the electron diffusion region, a flexible design for spacecraft separations, and a robust data retrieval scheme ensures that the science objective of the MMS mission will be achieved.

Acknowledgements The MMS mission design is a collaboration between the payload team and the GSFC Flight Dynamics group. The authors would like to thank the many men and women who worked on the mission design.

\section{References}

J.L. Burch et al., Magnetospheric multiscale overview and science objectives. Space Sci. Rev. (2014, this issue)

M.W. Dunlop, Q.-H. Zhang, Y.V. Bogdanova, K.J. Trattner, Z. Pu, H. Hasegawa, J. Berchem, M.G.G.T. Taylor, M. Volwerk, J.P. Eastwood, B. Lavraud, C. Shen, J.-K. Shi, J. Wang, D. Constantinescu, A.N. Fazakerley, H. Frey, D. Sibeck, P. Escoubet, J.A. Wild, Z.X. Liu, C. Carr, Magnetopause reconnection across wide local time. Ann. Geophys. 29, 1683 (2011)

J.P. Eastwood, T.D. Phan, M. Øieroset, M.A. Shay, Average properties of the magnetic reconnection ion diffusion region in the Earth's magnetotail: the 2001-2005 Cluster observations and comparison with simulations. J. Geophys. Res. 115, A08215 (2010). doi:10.1029/2009JA014962

D.H. Fairfield, A statistical determination of the shape and position of the geomagnetic neutral sheet. J. Geophys. Res. 85, 775 (1980)

H.U. Frey, T.D. Phan, S.A. Fuselier, S.B. Mende, Continuous magnetic reconnection at Earth's magnetopause. Nature 426, 533-537 (2003). doi:10.1038/nature02084

S.A. Fuselier, S.M. Petrinec, K.J. Trattner, Stability of the high-latitude reconnection site for steady northward IMF. Geophys. Res. Lett. 27, 473-476 (2000)

S.A. Fuselier, H.U. Frey, K.J. Trattner, S.B. Mende, J.L. Burch, Cusp aurora dependence on interplanetary magnetic field $B_{z}$. J. Geophys. Res. 107(A7), 1111 (2002). doi:10.1029/2001JA900165

S.A. Fuselier, S.M. Petrinec, K.J. Trattner, Anti-parallel magnetic reconnection rates at the Earth's magnetopause. J. Geophys. Res. 115, A10207 (2010). doi:10.1029/2010JA015302

S.A. Fuselier, W. Lewis, Properties of near-Earth magnetic reconnection from in-situ observations. Space Sci. Rev. 160, 95-121 (2011). doi:10.1007/s11214-011-9820-x

S.A. Fuselier, K.J. Trattner, S.M. Petrinec, Antiparallel and component reconnection at the dayside magnetopause. J. Geophys. Res. 116, A10227 (2011). doi:10.1029/2011JA016888

K. Genestreti, S.A. Fuselier, J. Goldstein, T. Nagai, An empirical model for the location and occurrence rate of near-Earth magnetotail reconnection. J. Geophys. Res. 118 (2013). doi:10.1002/2013JA019125

S.T. Griffiths, S.M. Petrinec, K.J. Trattner, S.A. Fuselier, J.L. Burch, T.D. Phan, V. Angelopoulos, A probability assessment of encountering dayside magnetopause diffusion regions. J. Geophys. Res. 116, A02214 (2011). doi:10.1029/2010JA015316

M. Hesse et al., Space Sci. Rev. (2014, this issue). doi:10.1007/s11214-014-0078-y

B.H. Mauk et al., Space Sci. Rev. (2014, this issue). doi:10.1007/s11214-014-0055-5

T. Nagai et al., Solar wind control of the radial distance of the magnetic reconnection site in the magnetotail. J. Geophys. Res. 110(A9), 011207 (2005). doi:10.1029/2005JA011207

R. Nakamura et al., Motion of the dipolarization front during a flow burst event observed by Cluster. Geophys. Res. Lett. 29 (2002). doi:10.1029/2002GL015763

T.K.M. Nakamura, R. Nakamura, A. Alexandrova, Y. Kubota, T. Nagai, Hall magnetohydrodynamic effects for three-dimensional magnetic reconnection with finitie width along the direction of the current. J. Geophys. Res. 117(A3), 017006 (2012). doi:10.1029/2011JA017006 
S.M. Petrinec, M.A. Dayeh, H.O. Funsten, S.A. Fuselier, D. Heirtzler, P. Janzen, H. Kucharek, D.J. McComas, E. Möbius, T.E. Moore, D.B. Reisenfeld, N.A. Schwadron, K.J. Trattner, P. Wurz, Neutral atom imaging of the magnetospheric cusp. J. Geophys. Res. 116, A07203 (2011). doi:10.1029/2010JA016357

T. Phan, H.U. Frey, S. Frey, L. Peticolas, S. Fuselier, C. Carlson, H. Reme, J.-M. Bosqued, A. Balogh, M. Dunlop, L. Kistler, C. Mouikis, I. Dandouras, J.-A. Sauvaud, S. Mende, J. McFadden, G. Parks, E. Moebius, B. Klecker, G. Paschmann, M. Fujimoto, S. Petrinec, M.F. Marcucci, A. Korth, R. Lundin, Simultaneous Cluster and IMAGE observations of cusp reconnection and auroral proton spot for northward IMF. Geophys. Res. Lett. 30(10), 1509 (2003). doi:10.1029/2003GL016885

T.D. Phan et al., IDS 1: establishing the contexts for reconnection diffusion region encounters and strategies for capturing and transmitting burst data in the diffusion regions. Space Sci. Rev. (2014, this issue)

C. Pollock et al., Fast plasma investigation for magnetospheric multiscale. Space Sci. Rev. (2014, this issue)

A. Runov, V. Angelopoulos, M.I. Sitnov, V.A. Sergeev, J. Bonnell, J.P. McFadden, D. Larson, K.-H. Glassmeier, U. Auster, THEMIS observations of an earthward-propagating dipolarization front. Geophys. Res. Lett. 36, L14106 (2009). doi:10.1029/2009GL038980

Science and Technology Definition Team, The Magnetospheric Multiscale mission-resolving fundamental processes in space plasmas. Report of the NASA Science and Technology Definition Team, NASA/TM2000-NASA/TM-209883, 1999

C. Schiff, E.J. Dove, Monte Carlo simulations of the formation flying dynamics for the Magnetospheric Multiscale (MMS) mission, in ISSFD Conference, Sao Paulos de Campos (2011)

D.G. Sibeck, R.E. Lopez, E.C. Roelof, Solar wind control of the magnetopause shape, location, and motion. J. Geophys. Res. 96, 5489 (1991)

C.R. Tooley, R.K. Black, P.D. Spidaliere, B.P. Robertson, G.T. Davis, N.E. Haghani, J.M. Stone, S.E. Pope, C. Pankratz, The magnetospheric multiscale constellation. Space Sci. Rev. (2014, this issue)

R.B. Torbert et al., The FIELDS instrument suite on MMS: scientific objectives, measurements, and data products. Space Sci. Rev. (2014, this issue)

K.J. Trattner, J.S. Mulcock, S.M. Petrinec, S.A. Fuselier, Location of the reconnection line at the magnetopause during southward IMF conditions. Geophys. Res. Lett. 34, L03108 (2007a). doi:10.1029/ 2006GL028397

K.J. Trattner, J.S. Mulcock, S.M. Petrinec, S.A. Fuselier, Probing the boundary between antiparallel and component reconnection during southward interplanetary magnetic field conditions. J. Geophys. Res. 112, A08210 (2007b). doi:10.1029/2007JA012270

K.J. Trattner, S.M. Petrinec, S.A. Fuselier, T.D. Phan, The location of reconnection at the magnetopause: testing the maximum shear model with THEMIS observations. J. Geophys. Res. 117, A01201 (2012). doi:10.1029/2011JA016959

N. Tsyganenko, Modeling the Earth's magnetospheric magnetic field confined within a realistic magnetopause. J. Geophys. Res. 100, 5599 (1995)

G.G. Wawrzyniak, J.R. Carpenter, D.J. Mattern, T.W. Williams, N.A. Ottenstein, B.A. Jones, Conjunction assessment concept of operations for the Magnetospheric Multiscale (MMS) mission, in AAS/AIAA Astrodynamics Specialist Conference, Hilton Head (2013)

T.W. Williams, Launch window analysis for the Magnetospheric Multiscale mission, AAS 12-255, in Proceedings of the AAS/AIAA Space Flight Mechanics Meeting, Jan 29-Feb 2, 2012, Charleston, SC. Advances in Astronautical Sciences Series, vol. 143 (2012)

M.K. Young et al., Hot plasma composition analyzer for the magnetospheric multi-scale mission. Space Sci. Rev. (2014, this issue) 